\title{
Properties of planets in binary systems ${ }^{\star}$ The role of binary separation
}

\author{
S. Desidera ${ }^{1}$ and M. Barbieri ${ }^{1,2}$ \\ 1 INAF - Osservatorio Astronomico di Padova, Vicolo dell' Osservatorio 5, 35122 Padova, Italy \\ e-mail: silvano.desidera@oapd.inaf.it \\ 2 Dipartimento di Fisica, Università di Padova, Italy
}

Received 29 August 2006 / Accepted 1 October 2006

\begin{abstract}
Aims. The statistical properties of planets in binaries were investigated. Any difference to planets orbiting single stars can shed light on the formation and evolution of planetary systems. As planets were found around components of binaries with very different separation and mass ratio, it is particularly important to study the characteristics of planets as a function of the effective gravitational influence of the companion.

Methods. A compilation of planets in binary systems was made; a search for companions orbiting stars recently shown to host planets was performed, resulting in the addition of two further binary planet hosts (HD 20782 and HD 109749). The probable original properties of the three binary planet hosts with white dwarfs companions were also investigated. Using this updated sample of planets in binaries we performed a statistical analysis of the distributions of planet mass, period, and eccentricity, fraction of multiplanet systems, and stellar metallicity for planets orbiting components of tight and wide binaries and single stars.

Results. The only highly significant difference revealed by our analysis concerns the mass distribution of short-period planets. Massive planets in short period orbits are found in most cases around the components of rather tight binaries. The properties of exoplanets orbiting the components of wide binaries are compatible with those of planets orbiting single stars, except for a possible greater abundance of high-eccentricity planets. The previously suggested lack of massive planets with $P>100$ days in binaries is not confirmed.

Conclusions. We conclude that the presence of a stellar companion with separation smaller than 100-300 AU is able to modify the formation and/or migration and/or the dynamical evolution history of giant planets while wide companions play a more limited role.
\end{abstract}

Key words. stars: planetary systems - stars: binaries: visual - stars: general - stars: individual: HD 20782, HD 109749, GL 86

\section{Introduction}

The study of the frequency of planets in binary systems ${ }^{1}$ and of the properties of these planets and of the binary systems themselves is very important to improve our knowledge of planet formation and evolution. On one hand, the frequency of planets in binary systems has a strong effect on the global frequency of planets, more than half of solar type stars being in binary or multiple systems (Duquennoy \& Mayor 1991). On the other hand, the properties of planets in binaries, and any difference to those of the planets orbiting single stars, would shed light on the effects caused by the presence of the companions.

The search for planets in binaries can follow two complementary approaches. The first one is to perform dedicated surveys looking for planets in binary systems. Several programs are currently in progress focusing on different types of binaries. The SARG planet search has been studying about 50 wide pairs with similar components (Desidera et al. 2006) since late 2000. A similar study was recently started by the Geneva group using HARPS. About 100 spectroscopic binaries with orbital periods between 1.5 and $100 \mathrm{yr}$ were monitored by

* Table 1 and Appendices A-C are only available in electronic form at http://www . aanda.org

${ }^{1}$ Some of the systems considered in this study are actually hierarchical triple systems. For simplicity, we refer to the whole sample as binaries.
Eggenberger et al. (2006a), while Konacki (2005a) is monitoring about 450 stars in binaries.

The second approach is to study the binarity of the hosts of planets discovered in general surveys, which include many binary stars in their lists in spite of some selection biases against them. Dedicated adaptive optics observations are required to reveal faint companions close to the star (e.g. Patience et al. 2002; Chauvin et al. 2006; Mugrauer et al. 2006a; see Table 1 for further references). However, the search for brighter companions at large separation can be performed by checking astronomical catalogs for nearby, comoving objects.

The first analysis of the properties of planets in binaries revealed differences with respect to those of planets orbiting single stars. Zucker \& Mazeh (2002) showed some difference in the period-mass relation. A more complete statistical analysis by Eggenberger et al. (2004), hereafter E04, confirms that massive planets in close orbits are mostly found in binaries. They also propose other possible peculiar characteristics of planets in binaries: low eccentricities for orbital periods shorter than 40 days and a lack of massive planets with periods longer than 100 days in binaries. The estimated significance of these latter features is less than $97 \%$, therefore requiring larger samples for confirmation.

The continuing observational efforts of planet searches and dedicated follow-up to study the characteristics of planet hosts lead to a significant increase of the total number of planets in 
binaries. We now know more than 40 planets in binaries or multiple systems, an increase by more than a factor of two with respect to the first compilation of the planets in binary systems assembled by E04 (19 planets in 15 systems $^{2}$ ).

The recent publication of the Catalog of Nearby Exoplanets by Butler et al. (2006), hereafter B06, provides an updated and homogeneous assembly of planet and stellar properties (excluding binarity). This work is a useful starting point for a statistical comparison of the properties of planets orbiting the components of multiple systems and single stars. In this paper we present such a discussion.

The outline of the paper is the following. In Sect. 2 the samples of planets in binaries and in single stars are defined; in Sect. 3 the statistical analysis of the properties of planets in binaries and single stars is presented. In Sect. 4 the results and their implications are discussed. Appendices A-C address specific issues relevant for individual cases: in Appendix A we present the results of the search for companions of stars recently shown to host planets, resulting in two further binaries (HD 20782 and HD 109749), whose properties are discussed is detail; in Appendix B we discuss the probable original properties (mass ratio, separation) of the three systems including white dwarf (hereafter WD) companions; in Appendix C we provide details about individual objects.

\section{An updated compilation of planets in binaries}

We assembled the properties of the planets in multiple systems and of the multiple system themselves (Table 1, only available in electronic form). For the planet hosts not included in the latest study of multiplicity of planet hosts (Raghavan et al. 2006, hereafter R06), we performed a search in existing catalogs of binaries ${ }^{3}$. Details are presented in Appendix A.

The planetary properties, as well as mass and metallicity of the planet hosts, were taken from B06. The binary parameters were taken from the references listed in the Table caption. As we are interested in evaluating the actual dynamical effects of the companions, we listed individual masses, derived using the mass-luminosity relations by Delfosse et al. (2000) or taken from the works cited in the caption. In a few cases, the complete binary orbit is available, making feasible a more in depth analysis of the possible formation and evolution of the planet (see e.g. Thebault et al. 2004, for $\gamma$ Cep). The last column lists the critical semiaxis for dynamical stability of planetary orbit derived using Eq. (1) of Holman \& Weigert (1999). When the full binary orbit is not available, this was calculated deriving the semimajor axis from the projected separation and adopting an eccentricity of 0.35 (Fischer \& Marcy 1992; Duquennoy \& Mayor 1991). Remarks on individual objects are reported in Appendix C, while the properties of the systems containing a white dwarf are discussed in Appendix B.

The comparison sample of planets in single stars is that of B06, excluding the objects in Table 1. The mass limit adopted by B06 $\left(m \sin i<24 M_{\mathrm{J}}\right)$ is different to those adopted in R06.

\footnotetext{
2 The radial velocity variations of one of the stars listed in E04 (HD 219542B) was shown to be due to the activity jitter of the star and not to a planet (Desidera et al. 2004).

3 Some planets have been announced after the submission of this paper and they are not considered in this study. One of them is orbiting a component of a wide binary (ADS 16402B; Bakos et al. 2006b). The binary separation is $1550 \mathrm{AU}$. The two transiting planets discovered by Collier Cameron et al. (2006) have companions candidates discovered using adaptive-optics imaging, whose physical association to the planet hosts needs confirmation.
}

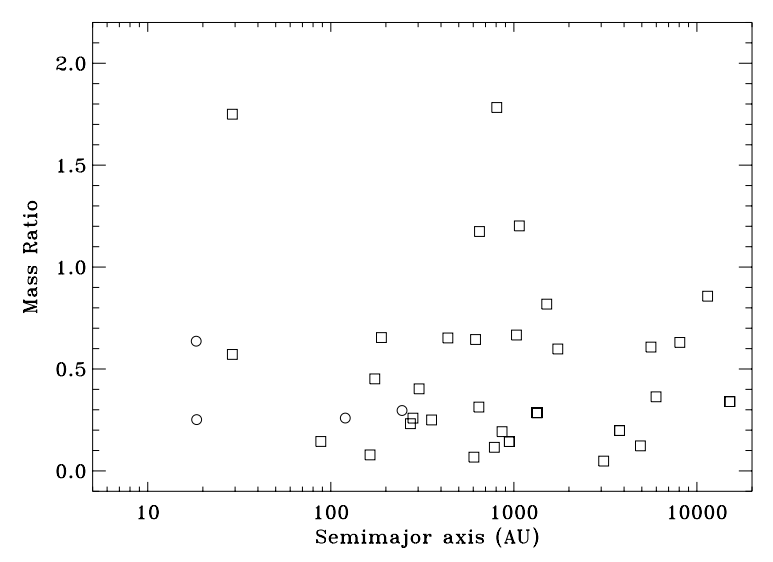

Fig. 1. Mass ratio vs. semimajor axis of the binary orbit for stars with planets in binary systems. Open circles represent the pairs for which binary orbit is available, open squares the pairs for which only the binary separation is available.

One "super-planet" in the range $m \sin i 13-24 M_{\mathrm{J}}$ was found in a binary, the companion orbiting HD 41004B.

\section{Statistical analysis}

Figure 1 shows the mass ratio vs. semimajor axis for stars with planets in multiple systems. For hierarchical triple systems in which the planet orbits the isolated companion, the masses of the binary companions to the planet host are summed. It appears that planets might exist in binaries with very different properties. In some cases (e.g. very low mass companions at a projected separation larger than $1000 \mathrm{AU}$ ) the dynamical effects of the companion on the formation and evolution of the planetary system might be very limited, while in the cases of very tight binaries the presence of the planet represents a challenge for the current models of planet formation (Hatzes \& Wuchterl 2005).

A simple look at Fig. 1 suggests a possible depopulated region for the binary semimajor axis between about 20 to $100 \mathrm{AU}$. A proper evaluation of its reality requires a detailed study of the selection effects of the original samples of radial velocity surveys and the discovery of stellar companions of planet hosts, which is postponed for a future study.

The dynamical effects of the companions on the circumstellar region of the planet hosts are certainly very different for the binaries in our sample. To consider the effects of dynamical perturbation by the stellar companion(s) we used the critical semiaxis for dynamical stability of the planet $a_{\text {crit }}$ (Holman $\&$ Wiegert 1999). This allows us to take into account the effects of both the separation and the mass ratio. The critical semiaxis for dynamical stability represents the limit for stable planetary orbits around the planet hosts (calculated for coplanar circular orbits). It is also similar to the radius of tidal truncation of the circumstellar disk (Pichardo et al. 2005; Pfahl \& Muterspaugh 2006). However, the area in which relative impact velocities between planetesimals is expected to allow planet formation might be significantly smaller than $a_{\text {crit }}$ (Thebault et al. 2006).

The critical semimajor axis $a_{\text {crit }}$ was used to divide the sample according to the relevance of the dynamical effects. We define as "tight" binaries those with $a_{\text {crit }}<75$ AU and "wide" binaries those with $a_{\text {crit }}>75 \mathrm{AU}$. The limit corresponds to a projected separation of about 200-300 AU depending on the mass ratio. The choice was fixed as a compromise, to obtain a sample of binaries wide enough to guarantee limited dynamical 


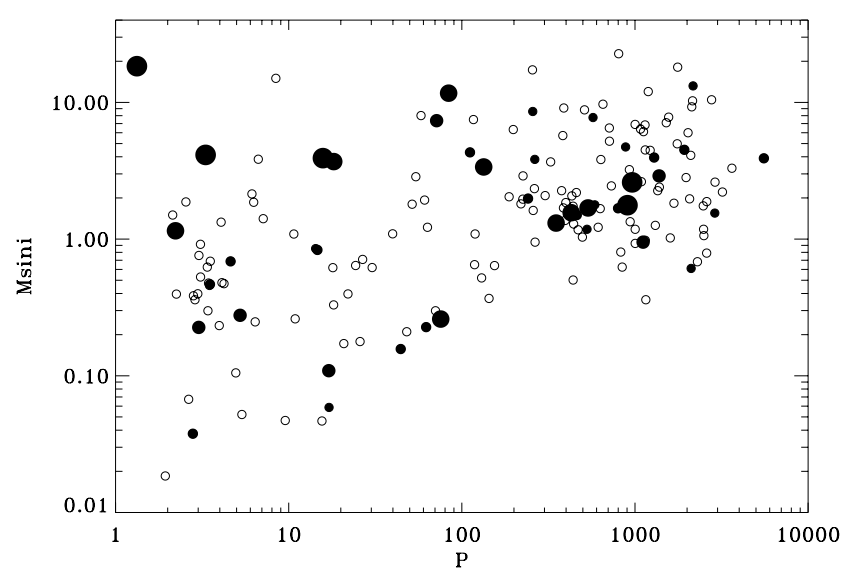

Fig. 2. Projected mass vs. orbital period of extrasolar planets. Open circles: single stars; filled circles: binary stars. The size of the symbol is proportional to the critical semimajor axis for dynamical stability (larger symbols refer to the tighter binaries).

perturbations for the portion of the disk on which the formation of giant planets is expected to occur and to allow the inclusion of an adequate number of objects in the "tight" binary sample. When relevant for the statistical analysis, the effect of changing this limit is considered in the following discussion.

The statistical comparison to test the hypothesis that the parameters of planets (mass, period, eccentricity) in tight and wide binaries and in single stars can be drawn from the same parent distribution was estimated using the Kolmogorov-Smirnov (hereafter KS) test and the Mann-Whitney U (hereafter MWU) test (Babu \& Feigelson 1996). We also used the hypergeometric distribution (see E04), that yields the probability of obtaining by chance a number of cases from a subsample of given size. The statistical analysis was performed including only planets with radial velocity (hereafter RV) semi-amplitude $K>15 \mathrm{~m} / \mathrm{s}$, to exclude planets with uncertain orbital parameters and to ensure a more homogeneous detectability. This choice has little effect on the results.

\subsection{The mass distribution of close-in planets}

Zucker \& Mazeh (2002) showed that planets in binaries follow a different mass period relation than those orbiting single stars. In particular, high-mass, short-period planets appear to be present only in binary systems. Such a correlation was confirmed by E04 but further inclusion of new systems makes it weaker (Mugrauer et al. 2005). The addition of further low-mass hot-Jupiters in binaries from our work consolidates this latter trend.

The possibility that the Zucker \& Mazeh correlation was simply due to the small number of planet in binaries known at that time can be considered. However, an alternative interpretation appears more convincing when one takes into account the binary properties of the massive hot-Jupiter hosts: these are mostly relatively tight binaries (Fig. 2). Indeed, Zucker \& Mazeh (2002) considered in their statistical analysis only binaries whose projected separation is smaller than $1000 \mathrm{AU}$, then excluding very wide binaries included in increasingly larger number in later studies.

To test this hypothesis statistically, we selected from Table 1 and B06 the planets with $P<40$ days (about in the middle of the so-called "period valley" on the period distribution of planets, see Udry et al. 2003 and Fig. 5). We performed a KS test
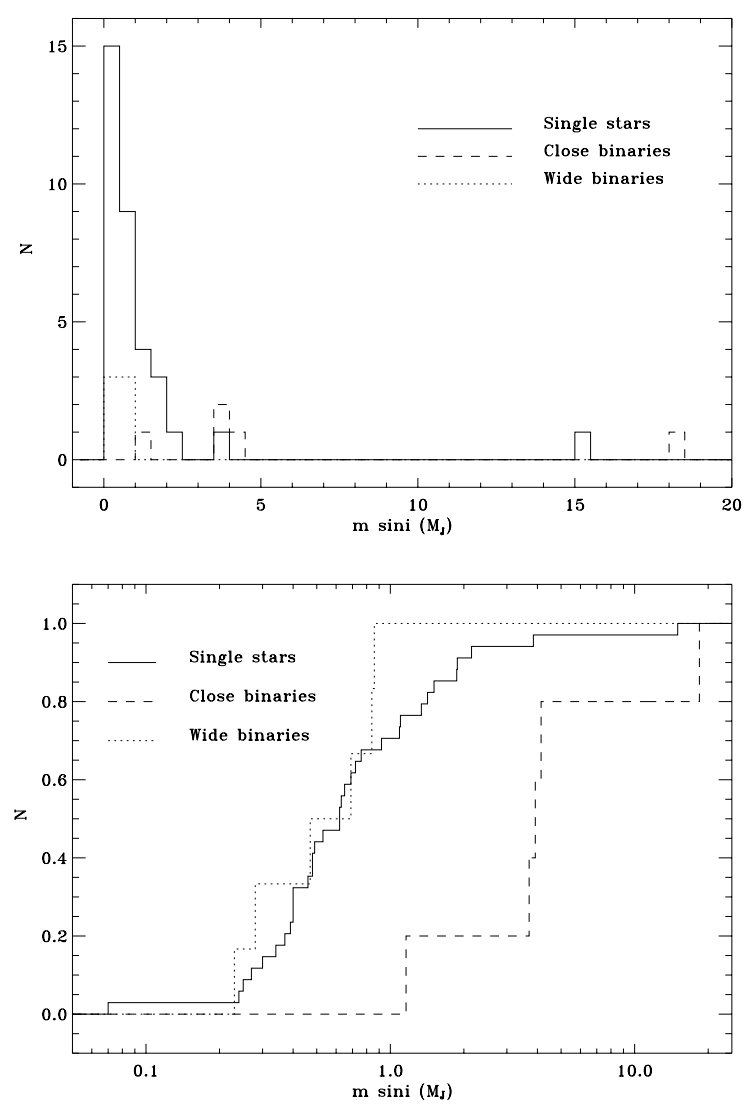

Fig. 3. Distribution of the projected mass $m \sin i$ for the planets with period shorter than 40 days and $K<15 \mathrm{~m} / \mathrm{s}$ orbiting single stars (continuous line), planets orbiting binaries with $a_{\text {crit }}<75 \mathrm{AU}$ (dashed line), and planets orbiting binaries with $a_{\text {crit }}>75 \mathrm{AU}$ (dotted line). Upper panel: histogram; lower panel: cumulative distribution.

comparing the mass distribution of planets with $P<40$ days orbiting single stars, binaries with $a_{\text {crit }}<75 \mathrm{AU}$ and binaries with $a_{\text {crit }}>75 \mathrm{AU}$ (34, 5, and 6 planets respectively) (Fig. 3). The hypothesis that the mass of close-in planets in tight binaries can be drawn from the same parent distribution of close-in planets orbiting members of wide binaries or single stars can be rejected with a probability of $98.2 \%$ and $99.5 \%$. The MWU test yields an even higher significance ( $>99.9 \%)$. The mass distribution of close-in planets orbiting single stars and wide binaries is not statistically different.

We checked the effects of the assumptions made on our analysis on the resulting significance. The significance of the difference of the mass distribution between planets in tight binaries and single stars is larger than $99.9 \%$ for $a_{\text {crit }}=50 \mathrm{AU}$, it is about $97.5 \%$ for $a_{\text {crit }}=100 \mathrm{AU}$ and becomes smaller moving the limit beyond $100 \mathrm{AU}$. The exclusion of super-planets more massive than $10 M_{\mathrm{J}}$ has some effect but the significance remains high ( $>99 \%$ ) for both tests. Changing the period limit of close-in planets in the range 20-200 days keeps the significance always larger than $99 \%$. The RV semi-amplitude selection limit plays little role.

For some of the close-in planets with $m \sin i>1.5 M_{\mathrm{J}}$ orbiting stars classified as single there are indications of the presence of significant dynamical perturbations. HD $118203 \mathrm{~b}$, HD 68988 b and HIP 14810 b have an unusually high eccentricity $(e=0.31,0.14$, and 0.15 respectively) for their orbital period ( 6 days). A second planet is orbiting HIP 14810 (B06), 

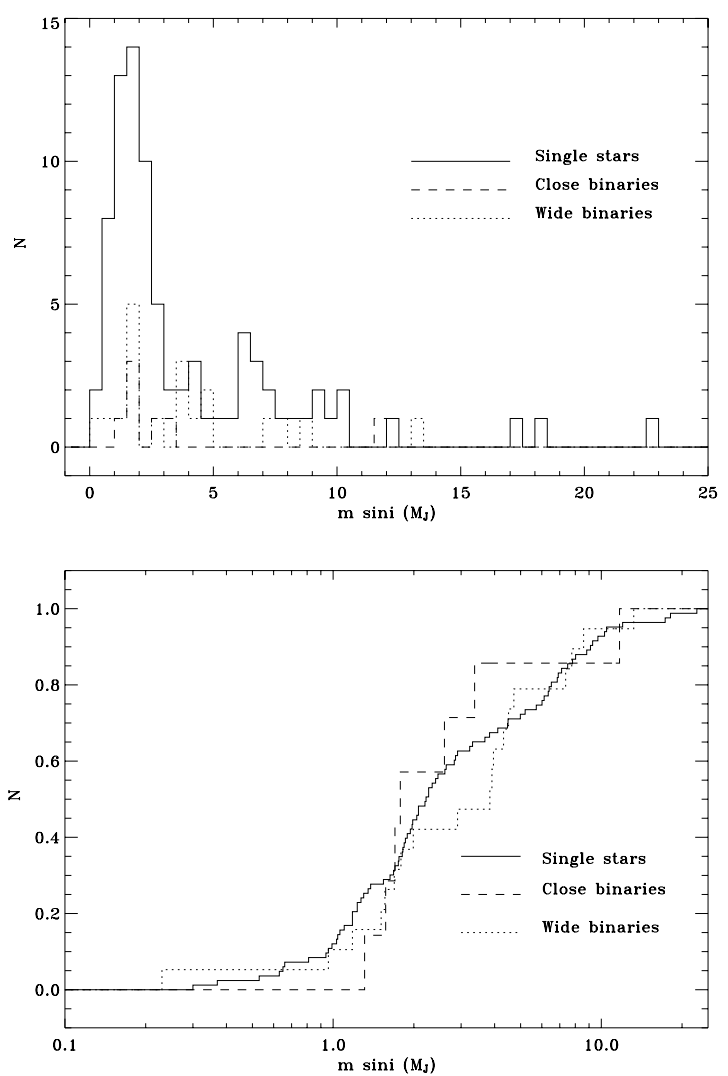

Fig. 4. Distribution of the projected mass $m \sin i$ for the planets with period longer than 40 days orbiting single stars (continous line), planets orbiting binaries with $a_{\text {crit }}<75 \mathrm{AU}$ (dashed line), and planets orbiting binaries with $a_{\text {crit }}>75 \mathrm{AU}$ (dotted line). Upper panel: histogram; lower panel: cumulative distribution.

while in the other two cases linear trends in the residuals from the short period orbit indicate the presence of a further companion in a long period orbit. Their mass and period will be revealed by the continuation of the observations, allowing a proper classification of their hosts as binary systems or multi-planet hosts. A candidate companion close to HD 162020 was reported by Chauvin et al. (2006), whose physical association remains to be established. For HD $73256 \mathrm{~b}$ there is currently no indication of additional companions.

\subsection{The mass distribution of planets in wide orbits}

Figure 4 shows the distribution of $m \sin i$ of planets with $P>$ 40 days. The lack of massive planets with $P>100$ days in binaries noted by E04 is no longer valid: three planets with $m \sin i \geq 5.0 M_{\mathrm{J}}$ are now in the sample. All of them are orbiting the components of very wide binaries (projected separation larger than $800 \mathrm{AU})$. The probability of having 3 planets with $P>100$ days and $m \sin i \geq 5.0 M_{\mathrm{J}}$ derived using the hypergeometric distribution is $17 \%$ when considering only wide binaries and $7.4 \%$ when considering all the binaries together. However, this probability becomes larger than $10 \%$ when considering a different mass limit (4-6 $M_{\mathrm{J}}$ ) and KS and MWU tests do not reveal statistically significant differences.

The mass distribution of long-period planets around tight binaries ( 7 planets) is not statistically significantly different with respect to that of planets in single stars and wide binaries (83 and 19 planets respectively).
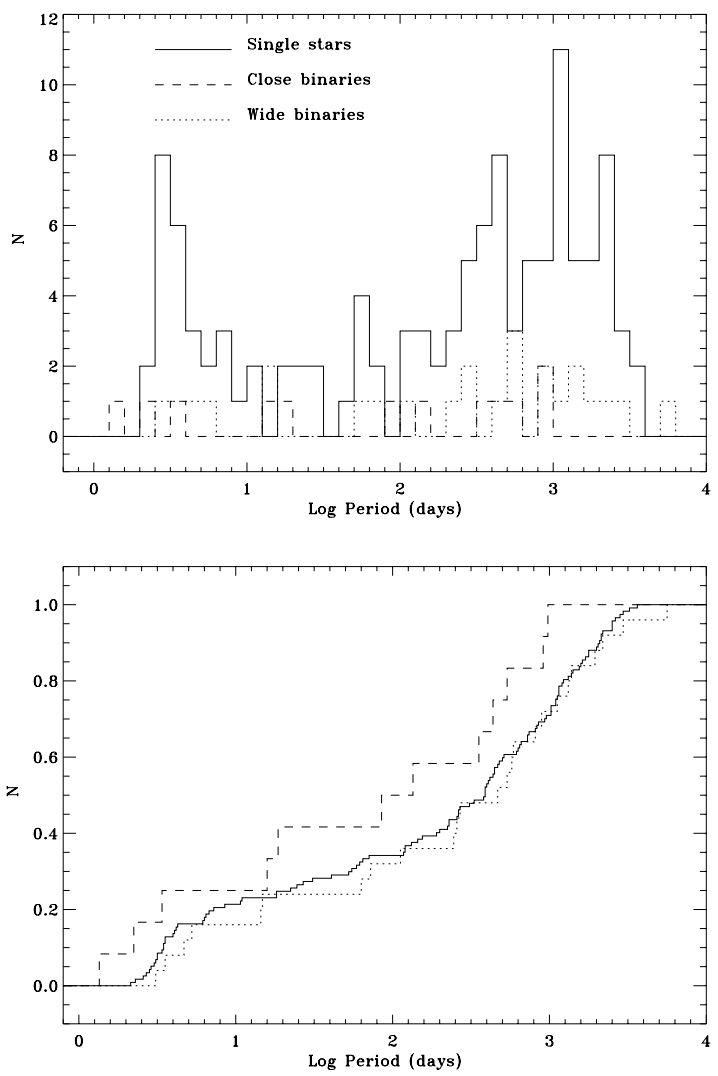

Fig. 5. Distribution of $\log$ (period) for the planets orbiting single stars (continous line), planets orbiting binaries with $a_{\text {crit }}<75 \mathrm{AU}$ (dashed line), and planets orbiting binaries with $a_{\text {crit }}>75 \mathrm{AU}$ (dotted line). Upper panel: histogram; lower panel cumulative distribution.

\subsection{The period distribution}

The period distribution of short period planets shows a pile-up for periods around 3 days (see e.g. B06). Only one of the five close-in planets in tight binaries has a period close to 3 days ( $\tau$ Boo b). Two have a very short period (HD 41004Bb and HD 189733b have the shortest and the third shortest period in the B06 catalog), while GL86b and HD 195019b have longer periods (15 and 18 days respectively). The differences are not statistically significant using KS and MWU tests.

When considering the period distribution of planets with $P>$ 40 days, there are hints that the period distribution of planets in tight binaries is different, with a lack of planets with periods longer than 1000 days. However, the KS and MWU tests do not show statistically significant differences.

When considering the whole period range (Fig. 5), the difference between the period distribution of planets around single stars and tight binaries (117 and 12 planets respectively) is more significant $(94.3 \%)$ for the MWU test while it remains not significant according to the KS (50\%). Similar values of significance are derived for the comparison between the period distribution of planets in tight vs. planets in wide binaries ( 25 planets). The discrepant level of significance between the two tests can be understood considering that the period distribution of planets in tight binaries appears to be shifted with respect to those orbiting single stars for the whole period range, but without very large deviations. The maximum difference of the cumulative distributions, the estimator used by the KS test, is then not significant, while the global shift is marginally significant by the 


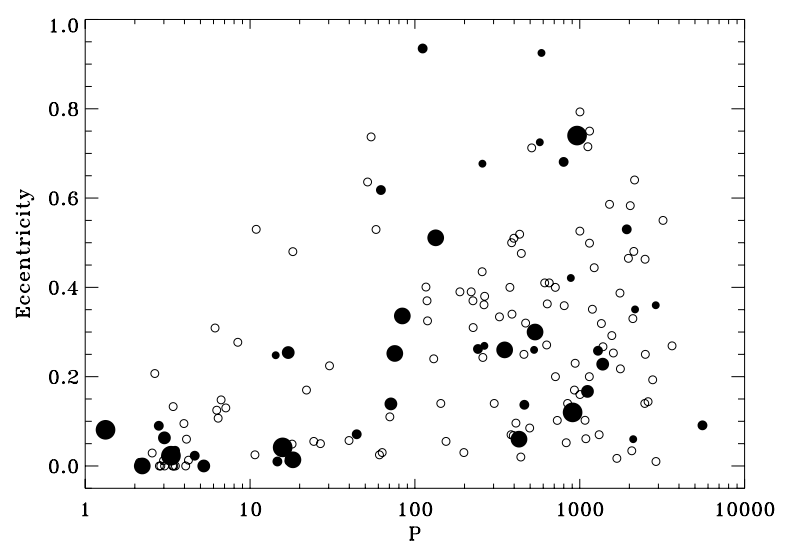

Fig. 6. Eccentricity vs. orbital period for planets in binaries (filled circles) and orbiting single stars (empty circles). Different sizes of filled circles refer to different periastron of the binary orbit (larger sizes: closer orbits).

MWU-test, that is sensitive instead to differences of the median of the distributions.

As the significance is not large and the different surveys have their own period sensitivity and biases concerning binarity (see Sect. 3.7), we conclude that the evidence of the different period distribution for planets orbiting single stars and components of tight binaries needs confirmation.

The period distribution of planets orbiting single stars and components of wide binaries are instead remarkably similar considering both separate period ranges and for the whole period range.

\subsection{The eccentricity distribution}

The possible link between high planet eccentricity and binarity has been considered since the discovery of the planet orbiting 16 Cyg B (Holman et al. 1997; Mazeh et al. 1997). It is thus relevant to consider the eccentricity distribution for planets orbiting tight and wide binaries and single stars.

Figure 6 shows the period-eccentricity diagram. The only two planets with $e>0.80$ (HD 80606b and HD 20782b) are in binary systems. However, planets with $e$ up to 0.75 appear to be present also around stars currently known as single.

The eccentricity distribution of planets in tight binaries with periods longer than 40 days (Fig. 7) is not significantly different to those orbiting single stars ${ }^{4}$.

The comparison for planets in wide binaries indicates no significant differences according to the KS test (74\%), while the MWU test gives a significance of $91.2 \%$ that the median of the two samples are different.

E04 noted that all the five planets in binaries in their sample with periods shorter than 40 days have an unusually low eccentricity $(e<0.05)$. They estimated a chance probability of $3.8 \%$ using the hypergeometric distribution. While a tendency to a low eccentricity of short-period planets in binaries seems to be still present ( 4 planets of 5 and 4 of 6 with $e<0.05$, for the tight and wide binaries respectively, compared with 17 of 34 for planets in single stars) a similar analysis in our sample (taking together wide and tight binaries) yields a probability of $12 \%$, therefore not confirming the E04 result at a highly significant level. KS and MWU tests also do not suggest statistically significant

\footnotetext{
${ }^{4}$ The large eccentricity of HD 41004Ba is uncertain $(e=0.74 \pm 0.20)$.
}
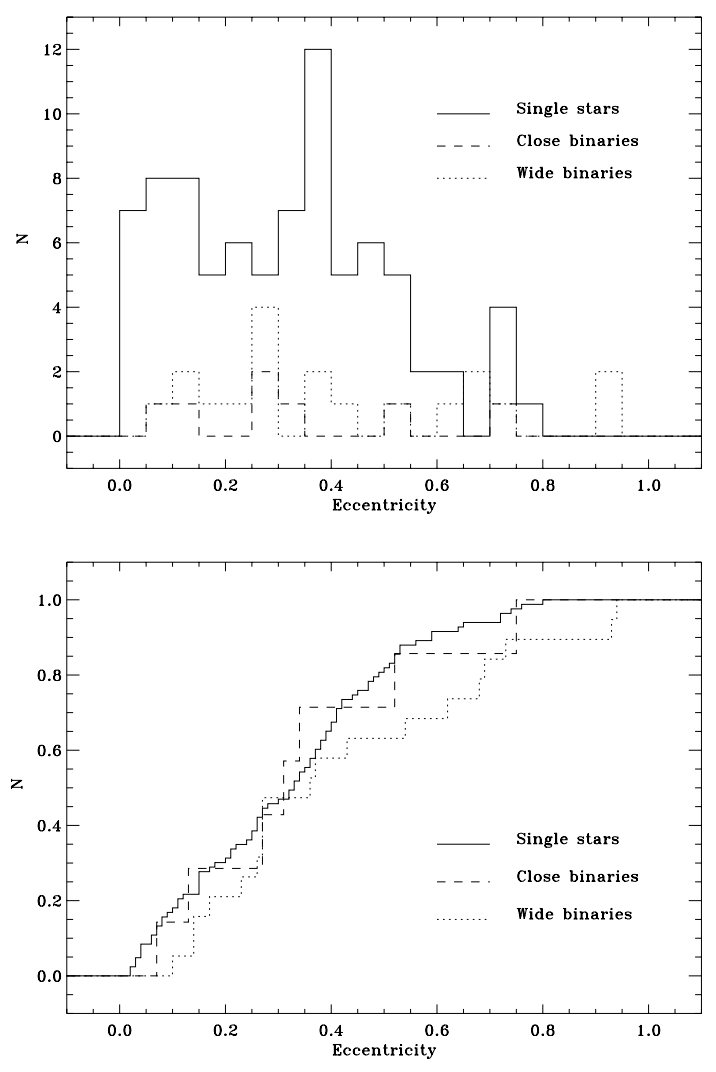

Fig. 7. Cumulative distribution of the planet eccentricity for the planets with period longer than 40 days orbiting single stars (continous line), planets orbiting binaries with $a_{\text {crit }}$ smaller than $75 \mathrm{AU}$ (dashed line), and planets orbiting binaries with $a_{\text {crit }}$ larger than $75 \mathrm{AU}$ (dotted line).

differences. Selecting only planets with periods between 10 and 40 days (to exclude objects whose orbits are circularizated by tidal effects) does not increase the significance of a lower eccentricity for short-period planets in binaries.

\subsection{Multi-planet systems}

The occurrence of systems with more than one planet around the components of wide binaries ( 4 of $23,17.3 \pm 12.3 \%$ ) is similar with respect to that of planets orbiting single stars (15 of 112, $13.3 \pm 4.7 \%)$. On the other hand, no multiple planets have been yet discovered around the components of tight binaries (13 objects). The closest binary with a known multi-planet system is $v$ And (projected separation $750 \mathrm{AU}$ ). However, the small number of tight binaries in the sample makes the lack of multi-planet systems not highly significant (probability of $15 \%$ of occurring by chance using the hypergeometric distribution). The similar fraction of multiplanet systems around single stars and wide companions is a further indication that the presence of a wide companion does not alter too much the process of planet formation.

\subsection{Metallicity of planets in binary systems}

The study of the metallicity of planet host in binaries can shed light on possible different planet formation mechanisms (see Sect. 4). The close-binary planet-hosts appears slightly more metal poor than single stars, while the wide-binary planethosts are slightly more metal rich. The comparison of cumulative distributions (Fig. 8) using KS and MWU tests indicates 

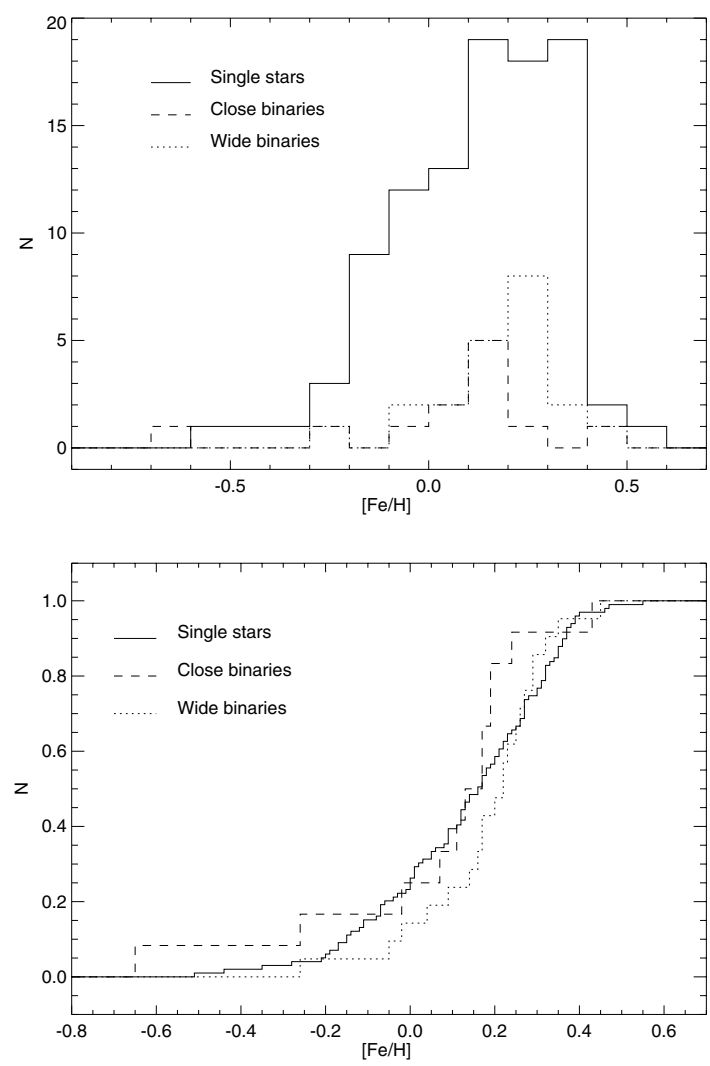

Fig. 8. Distribution of the stellar metallicity $[\mathrm{Fe} / \mathrm{H}]$ for the planets orbiting single stars (continous line), planets orbiting binaries with $a_{\text {crit }}<75 \mathrm{AU}$ (dashed line), and planets orbiting binaries with $a_{\text {crit }}>$ 75 AU (dotted line). Upper panel: histogram; Lower panel: cumulative distribution.

probabilities of $12.1 \%$ and $4.6 \%$ respectively that the metallicity of close and wide binary planet hosts can be drawn from the same parent population. The differences with respect to single stars are less significant (probability of $23.3 \%$ and $17.8 \%$ for the close binary vs. single stars and and wide binary vs. single stars respectively using the MWU test, a smaller significance using KS). Considering together all the binaries makes any difference not significant.

While intriguing, the possible differences of the metallicity of binary planet hosts should be taken with caution. Beside the rather large probability of a chance result, it should be considered that the small number of planets in close binaries makes the role of individual objects critical (e.g. HD 114762, whose companion might be a brown dwarf or even a low mass star, Cochran et al. 1991). Furthermore, indirect selection effects might cause spurious correlations (see Sect. 3.7).

\subsection{Selection effects and caveats}

The B06 catalog includes planets discovered by several different surveys. Different RV surveys typically have their own biases concerning binarity and other properties (e.g. stellar mass and evolutionary phase, metallicity, etc.). Furthermore, they have different precision and time spans, and thus different planet mass and period sensitivities.

All these effects cannot controlled in our study, and we cannot exclude that they play a role in producing some of the marginal differences we showed (e.g. in metallicity and the period distributions). However, the most significant result, the different mass distribution of close-in planets in tight binaries, should not be affected by these selection effects. In fact, these planets are the easiest to discover for any survey because of their short periods and large radial velocity amplitude.

An additional caveat is represented by the uncertainty in the upper limit of planetary mass, which is assumed in this study to be $24 M_{\mathrm{J}}$ following B06, and by the other choices performed in the analysis (e.g. period limits, etc.)

The sample of binaries among planet hosts is certainly not complete. A large fraction of the planet hosts were scrutinized only for comoving companions at rather large separations (a few arcsec). The analysis was performed considering the actual binary configuration that in some cases can be significantly different to that at the time of the formation of the planets. In the case of binary systems including a white dwarf, the probable original configuration can be derived estimating the original mass of the (now) white dwarf (see Appendix B), but other cases are speculative (see Appendix A.1).

\section{Discussion}

Exploiting the continuously expanding samples of extrasolar planets, we investigated the occurrence of a difference between planets in binaries and planets orbiting single stars. The size of the sample allowed us to divide the binaries in two sub-groups, "tight" and "wide" binaries. The separation was fixed at a critical semiaxis for dynamical stability equal to $75 \mathrm{AU}$. This corresponds to separations of about 200-300 AU depending on the mass ratio between the components.

\subsection{The properties of planets in tight binaries}

The most significant feature we were able to show is that the mass distribution of close-in planets around close and wide binaries is different. Only the close-in planets in tight binaries have a mass distribution significantly different to those orbiting single stars. This result implies that the formation and/or migration and/or dynamical evolution processes acting in the presence of a sufficiently close external perturber are modified with respect to single stars.

The fact that the hosts of massive planets in close orbit are typically binaries somewhat resembles the results by Tokovinin et al. (2006) i.e. short-period spectroscopic binaries have in most cases a further companion. That a third member could act as an angular momentum sink resulting in a closer inner binary was first suggested by Mazeh \& Shaham (1979). Tokovinin et al. (2006) favor generation of high orbital eccentricity by Kozai resonances, and subsequent tidal circularization, as the mechanism to explain the close spectroscopic binaries and their link with the presence of additional companions.

While it cannot be excluded that some of the massive closein planets in binaries were moved into their current location by Kozai migration, some other massive planets (GL $86 \mathrm{~b}$ and HD $195019 b$ ) have periastrons of 0.108 and 0.137 AU, probably excluding the occurrence of tidal circularization. This calls for a more general process to explain the presence of massive close-in planets.

A possible effect on migration and mass accumulation timescales in a disk in the presence of a companion was predicted by the models by Kley (2000). He considers the evolution of a Jupiter mass planet embedded in a circumstellar disk in binary systems with a separation of 50-100 AU, with eccentricity $e_{\text {bin }}=0.5$, and stellar masses of 1.0 and $0.5 M_{\odot}$. The 
presence of the companion significantly enhances the growth rate and make the migration timescale of the planet shorter. As discussed by E04, this can qualitatively explain the presence of massive close-in planets in binary systems. A step forward here is that we have shown that most of the binary systems that host the "anomalous" massive, close-in planets are indeed not too different to those modeled by Kley (2000), while E04 were forced to consider also binary systems with very wide separation, for which the described model is likely not to work. The models by Kley (2000) have as an initial condition an already formed planet and thus do not include the formation phase of giant planets. As discussed by Thebault et al. (2006), a possible way to achieve the formation of planets in a swarm of planetesimals perturbed by a stellar companion might be type II runaway accretion identified by Kortenkamp et al. (2001). More detailed modeling is required to achieve a full understanding of the processes that cause the differences of planet properties in tight binaries.

Additional possible differences of the properties of planets in tight binaries (to be confirmed as the statistical significance is not very high and selection effects might be at work) are the different period distributions (there are no planets with $P>1000$, the close-in planets probably do not pile-up at period close to three days) and the lack of multi-planet systems.

In a few cases, the lack of long period planets can be understood as the result of dynamical stability constrains: for $\gamma$ Cep and HD 41004A, the critical semimajor axis for dynamical stability is just 1.9 and $\sim 3$ times the planet semimajor axis. For the other cases, a more general mechanism should be at work (enhanced migration; Kley 2000 or lack of suitable conditions for planet formation in the external parts of the disks, under the gravitational influence of the companion).

The fact that properties of planets in tight binaries are different to those orbiting single stars can be exploited to distinguish between different scenarios for their origin. As discussed by e.g. Hatzes \& Wurchterl (2005) and Thebault et al. (2004), the presence of planets in binaries with a separation of $20 \mathrm{AU}$ or less represents a challenge for our current view of planet formation. Such problems can be overcome if the binary was initially wider, and its orbit was modified through dynamical interactions in star clusters after planet formation (Pfahl \& Muterspaugh 2006). The different mass distribution of planets in close binaries implies that, if this is the case, the dynamical interactions should not only modify the binary orbit but also force a massive planet in external orbit to migrate inward, as massive hot Jupiters are rare around single stars. This point should be addressed by future dynamical simulations. Kozai oscillations and tidal circularization after the dynamical interaction cannot be excluded in some cases (large relative inclinations between the planet and binary orbits might be the result of the proposed dynamical encounters). However, less massive planets originally in external orbits should follow a similar fate and thus we expect that low mass planets should also be present in close orbits in tight binaries, at odds with current observational data. Furthermore, as discussed above, Kozai migration cannot explain planets with periods longer than a few days and low eccentricities.

Further clues to the origin of planets in tight binaries can be derived by a determination of their frequency. Pfahl \& Muterspaugh (2006) predict that the fraction of close binaries ${ }^{5}$ that dynamically acquire giant planets is about $0.1 \%$, with an uncertainty of about one order of magnitude. They note that the

5 The definition of close binaries used by Pfahl \& Muterspaugh (2006) (semimajor axis less than $50 \mathrm{AU}$ ) is different to that adopted here. rough number of these planets seems too high to be compatible with these predictions. However, the number of tight binaries included in the radial velocity samples is not well known. A more detailed analysis is postponed to a forthcoming study.

The mentioned difficulties of the binary-interaction scenario to account for the occurrence of planets in close binaries leave open the alternative hypothesis, i.e. giant planets formed in binaries with small separation at the time of planet formation, possibly in a different way than planets around single stars.

The metallicity distribution of tight binaries with planets can give clues to the formation mechanism for these planets. The large effect of the stellar metallicity on the frequency of planetary companions is well established (Fischer \& Valenti 2005; Santos et al. 2004) and can be understood in the framework of the core-accretion model for the formation of giant planets (Ida \& Lin 2004; Robinson et al. 2006). If these planets formed in a different way than those orbiting single stars and the components of wide binaries, e.g. by disk instability triggered by dynamical perturbations (Boss 2006), we should expect a lower metallicity for planet hosts in close binaries, as the disk instability mechanism is expected to be rather insensitive to metallicity (Boss 2002). There is a marginal indication of a larger fraction of metal poor stars among tight binaries with planets. However, the distribution of metallicity remains on average larger than that if the solar vicinity, with a mean and median larger than the solar value. This indicates that high metallicity is a factor favoring planet formation also in tight binary systems.

\subsection{The properties of planets in wide binaries}

The mass and period distributions of planets in wide binaries are not statistically significant different to those of planets orbiting single stars. The lack of long-period massive planets in binaries shown by E04 is not confirmed at a highly significant level in our study. The fraction of multi-planet systems is similar for planets orbiting single stars and components of multiple systems. These results indicate that a distant companion (separation $>300-500 \mathrm{AU}$ ) does not significantly affect the process of planet formation. The only marginally significant difference between planets orbiting single stars and components of wide binaries concerns the planet eccentricity.

\subsection{Is binarity the cause of the high eccentricities ?}

The eccentricity distribution of planets in wide binaries shows a marginal excess of high-eccentricity $(e \geq 0.6)$ planets. However, current results indicate that the high planet eccentricity is not confined to planets in binaries, and that the possible differences in eccentricity are limited to the range $e \geq 0.5-0.6$. This indicates that there are mechanism(s) generating planet eccentricity up to $0.4-0.5$ that are independent of the binarity of the planet host, and are characteristic of formation and evolution of a planetary system (e.g. disk-planet interactions: Tremaine \& Zakamska 2004; planet-planet scattering: Marzari \& Weidenschilling 2002; Ford et al. 2003). These probably act during or shortly after planet formation. Further eccentricity enhancements, possibly linked to the presence of a companion, might take place at later epochs. In fact, Takeda et al. (2006) noted that most higheccentricity planets orbit old stars (ages $>5$ Gyr). Mechanisms that require long time scales to modify planetary orbits then seem favored.

A popular mechanism that might produce high eccentricity planets in binaries is represented by Kozai oscillation. Kozai 
oscillation is expected to be at work also for low mass companions at large separation (the case of most of the companions of planet hosts discovered up to now), provided that a sufficiently long time is available and that the planet has a sufficiently long period to prevent the suppression of Kozai oscillation by general relativistic effects (Takeda \& Rasio 2005; Wu \& Murray 2003). As an example, a brown dwarf companion such as HD 3651B (mass about $0.06 M_{\odot}$, projected separation about $500 \mathrm{AU}$ ) might be able to induce the eccentricity oscillation for a planet with an initial semimajor axis larger than about 3 AU.

Kozai oscillations are expected to produce large planet eccentricities. Wu \& Murray (2003) considered in detail the possibility that the high eccentricity of HD 80606b $(e=0.93)$ is due to Kozai oscillation. This was found to be possible only for a relative inclination of the planetary and binary orbit larger than $85 \mathrm{deg}$. In the case of the only other planet with $e>0.8$ (HD 20782b), the period of the Kozai eccentricity oscillation derived from Eq. (2) of Takeda \& Rasio (2005) is much longer than the Hubble time. Therefore, the extremely high eccentricities of HD 20782b is unlikely to be due to the Kozai mechanism (see Appendix A.1), unless further companions exist in the system at smaller separation.

An alternative to Kozai oscillations is represented by chaotic evolution of planetary orbits induced by dynamical perturbations (Benest \& Gonczi 2003; Marzari et al. 2005). This kind of evolution might also arise for the dynamical interactions within the planetary system, without requiring a stellar companion in a wide orbit.

To further investigate the origin of the high eccentricities, we consider the eccentricity distributions of planets residing in multiplanet systems and isolated planets (Fig. 9). There is a lack of high eccentricity planets in multi-planet systems (as noted also by Takeda et al. 2006). When considering planets with $P>40$ days, the comparison of the two distributions reveals a difference that is significant to about a $89.1 \%$ confidence level (according to the MWU test), similar to the results of the planets in wide binaries vs those in single stars. This can be understood considering that planetary systems with highly eccentric planets are in general more subject to close encounters, and then less stable, than a system populated with planets in low eccentricity orbits. If the chaotic evolution of a planetary system has as a final result a planet on a highly eccentricity orbit, it is probable that that planet remains alone. On the other hand, Kozai oscillations are more likely to take place in single-planet systems, as they are suppressed in the presence of significant mutual planet-planet interactions. Therefore, eccentricity enhancements due to Kozai oscillation are expected to occur for single-planets systems with a companion in a suitable binary configuration. A proper evaluation of the link between high eccentricities, binarity of planet hosts, and planet multiplicity requires larger samples to allow more statistically significant inferences.

\section{Conclusion}

The only highly significant difference shown in our analysis concerns the mass distribution of short-period planets in tight binaries. Massive planets in short period orbit are found in most cases around the components of rather tight binaries. Other possible peculiar features of planets in tight binaries with respect to planets orbiting single stars, such as a lack of long-period planets and multiple planets, are of marginal significance and need confirmation.

The properties of exoplanets orbiting the components of wide binaries are compatible with those of planets orbiting
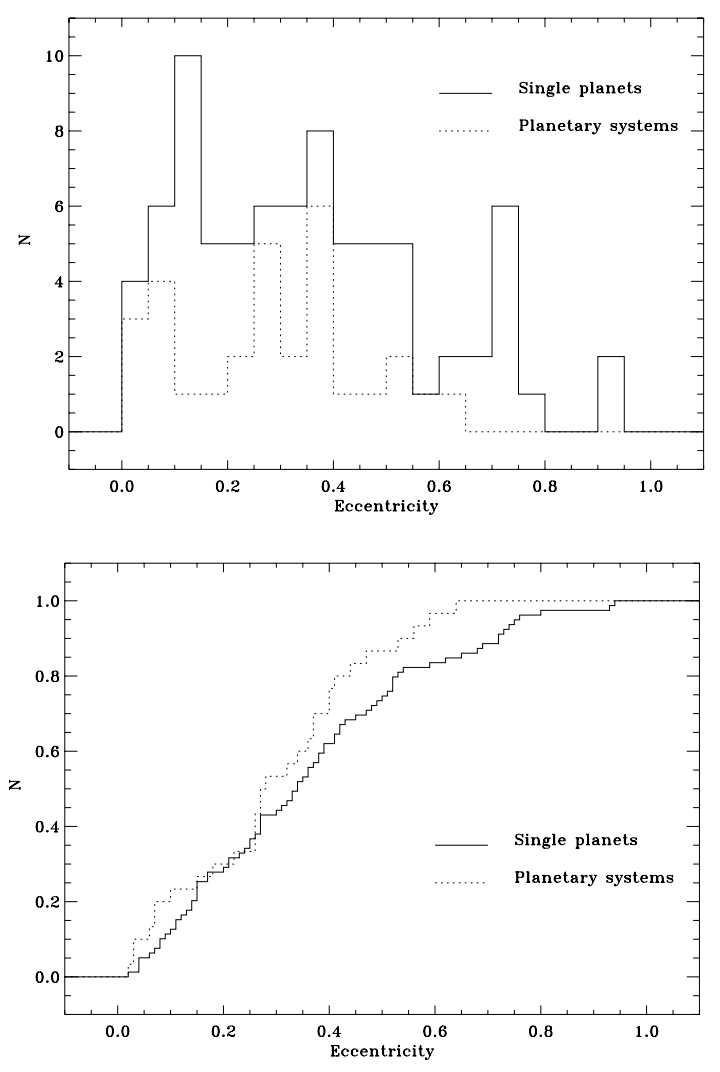

Fig. 9. Distribution of the planet eccentricity for the single planets (continous line), and planets in planetary systems (dotted line). Upper panel: histogram; lower panel: cumulative distribution.

single stars, except for a possible greater abundance of planets on highly eccentric orbits. The previously suggested lack of massive planets with $P>100$ days in binaries is not confirmed.

This result indicates that the binary separation plays a role in affecting the properties of planets and that the simple classification of planets in binaries vs planets orbiting single stars is not adequate to describe the dynamical effects. This opens the perspective for more detailed modeling of the role of stellar companions on the formation and evolution of planetary systems.

Acknowledgements. This research has made use of the SIMBAD database, operated at CDS, Strasbourg, France, and of data products from the Two Micron All Sky Survey. We thank J.R. Hurley for providing the BSE program. We thank G. Takeda for useful information about the stellar ages of planet hosts. We thank R. Gratton for his comments and suggestions. We thank the anonymous referee for a prompt and detailed report. This work was funded by COFIN 2004 "From stars to planets: accretion, disk evolution and planet formation" by the Ministero Univ. e Ricerca Scientifica Italy.

\section{References}

Aitken, R. G. 1932, New general catalogue of double stars within 120 of the North pole, Carnegie Inst. Washington Pub. n. 417

Allende Prieto, C., Barklem, P. S., Lambert, D. L., \& Cunha, K. 2004, A\&A, 420,183

Babu, G. J., \& Feigelson, E. D. 1996, Astrostatistic, 67

Bakos, G., Pal, A., Latham, D., Noyes, R. W., \& Stefanik, R. P. 2006a, ApJ, 641, L57

Bakos, G., Noyes, R. W., Kovacs, G., et al. 2006, ApJ, submitted [arXiv: astro-ph/0609369]

Bedin, L. R., Salaris, M., Piotto, G., et al. 2005, ApJ, 624, L45

Benest, D., \& Gonczi, R. 2003, Earth, Moon and Planets, 93, 175

Bensby, T., Feltzing, S., Lundstrom, I., \& Ilyin, I. 2005, A\&A, 433, 185

Bessell, M. S. 2000, PASP, 112, 961

Boss, A. P. 2002, ApJ, 567, L149 
Boss, A. P. 2006, ApJ, 641, 1148

Busso, M., Gallino, R., \& Wasserburg, G. J. 1999, ARA\&A, 37, 239

Butler, R. P., Wright, J. T., Marcy, G. W., et al. 2006, ApJ, 646, 505 (B06)

Castro, S., Porto De Mello, G. F., \& Da Silva, L. 1999, MNRAS, 305, 693

Chauvin, G., Lagrange, A.-M., Udry, S., et al. 2006, A\&A, 456, 1165

Cochran, W. D., Hatzes, A. P., \& Hancock, T. J. 1991, ApJ, 380, L35

Collier Cameron, A., Bouchy, F., Hebrard, G., et al. 2006, MNRAS, in press [arXiv:astro-ph 0609688]

Cutri, R. M., Skrutskie, M. F., Van Dyk, S., et al. 2003, Explanatory Supplement to the 2MASS All Sky Data Release (www . ipac. caltech. edu/2mass)

Delfosse, X., Forveille, T., Segransan, D., et al. 2000, A\&A, 364, 217

Desidera, S., Gratton, R. G., Endl, M., et al. 2004, A\&A, 420, L27

Desidera, S., Gratton, R. G., Claudi, R. U., et al. 2006, Proc. of the conference Tenth Anniversary of 51 Peg-b: status of and prospects for hot Jupiter studies, 119

Dommanget, J., \& Nys, O. 2002, Catalogue of the components of Double and Multiple Stars (CCDM), Observations et Travaux 54, 5

Duquennoy, A., \& Mayor, M. 1991, A\&A, 248, 485

Ecuvillon, A., Israelian, G., Santos, N. C., et al. 2006, A\&A, 445, 633

Eggenberger, A., Mayor, M., \& Udry, S. 2004, A\&A, 417, 353 (E04)

Eggenberger, A., Udry, S., \& Mayor, M., et al. 2006a, in ESO Workshop on Multiple Stars Across the HR Diagram, in press

Eggenberger, A., Mayor, M., Naef, D., et al. 2006b, A\&A, 447, 1159

Els, S., Sterzik, M. F., Marchis, F., et al. 2001, A\&A, 370, L1

ESA 1997, The Hipparcos and Tycho Catalogues, ESA SP-1200

Everhart, E. 1985, ASSL Vol. 115: IAU Colloq., 83, 185

Fischer, D. A., \& Marcy, G. W. 1992, ApJ, 396, 178

Fischer, D., \& Valenti, J. 2005, ApJ, 622, 1102

Fischer, D. A., Laughlin, G., Marcy, G. W., et al. 2006, ApJ, 637, 1094

Ford, E. B., Rasio, F. A., \& Yu, K. 2003, in Scientific Frontiers in Research on Extrasolar Planets, ASP Conf. Ser., 294, 181

Gilli, G., Israelian, G., Ecuvillon, A., Santos, N. C., \& Mayor, M. 2006, A\&A, 449,723

Girardi, L., Bertelli, G., Bressan, A., et al. 2002, A\&A, 391, 195

Gray, R. O., Corbally, C. J., Garrison, R. F., et al. 2006, AJ, 132, 161

Hale, A. 1994, AJ, 107, 306

Hatzes, A. P., Cochran, W. D., Endl, M., et al. 2003, ApJ, 599, 1383

Hatzes, A. P., \& Wuchterl, G. 2005, Nature, 436, 182

Hauser, H., \& Marcy, G. W. 1999, PASP, 111, 321

Holman, M., Touma, J., \& Tremaine, S. 1997, Nature, 386, 254

Holman, M. J., \& Wiegert, P. A. 1999, AJ, 117, 621

Hurley, J. R., Tout, C. A., \& Pols, O. R. 2002, MNRAS, 329, 897

Ida, S., \& Lin, D. N. C. 2004, ApJ, 616, 567

Jeffries, R. D., \& Smalley, B. 1996, A\&A, 315, L19

Jones, H. R. A., Butler, R. P., Tinney, C. G., et al., MNRAS, 369, 249

King, J. R., Villarreal, A. R., Soderblom, D. R., Gulliver, A. F., \& Adelman, S. J. 2003, AJ, 125, 1980

Kley, W. 2000, IAU Symp., 211

Konacki, M. 2005a, ApJ, 626, 431

Konacki, M. 2005b, Nature, 436, 230

Kortenkamp, S., Wetherill, G., \& Inaba, S. 2001, Science, 293, 1127

Lagrange, A.-M., Beust, H., Udry, S., Chauvin, G., Mayor, M. 2006, A\&A, 459, 955

Lowrance, P. J., Kirkpatrick, J. D., \& Beichman, C. A. 2002, ApJ, 572, L79
Marzari, F., Weidenschilling, S. J. 2002, Icarus, 156, 570

Marzari, F., Weidenschilling, S. J., Barbieri, M., \& Granata, V. 2005, ApJ, 618, 502

Mason, B. D., Wycoff, G. L., Hartkopf, W. L., Douglass, G. G., \& Worley, C. E. 2001, AJ, 122, 3466 (WDS)

Mayor, M., Udry, S., Naef, D., et al. 2004, A\&A, 415, 391

Mazeh, T., \& Shaham, J. 1979, A\&A, 77, 145

Mazeh, T., Krymolowski, Y., \& Rosenfeld, G. 1997, ApJ, 477, L103

Monet, D. G., Levice, S. E., Casian, B., et al. 2003, AJ, 125, 984

Mugrauer, M., \& Neuhauser, R. 2005, MNRAS, 361, L15

Mugrauer, M., Neuhauser, R., Mazeh, T., Guenther, E., \& Fernandez, M. 2004a, $\mathrm{AN}, 325,718$

Mugrauer, M., Neuhauser, R., Mazeh, T., Alves, J., \& Guenther, E. 2004b, A\&A, 425,249

Mugrauer, M., Neuhauser, R., Seifahrt, A., Mazeh, T., \& Guenther, E. 2005, A\&A, 440, 1051

Mugrauer, M., Neuhauser, R., Mazeh, T., et al. 2006a, AN, 327, 321

Mugrauer, M., Seifahrt, A., Neuhauser, R., \& Mazeh, T. 2006b, MNRAS, 373, L13

Nordstrom, B., Mayor, M., Andersen, J., et al. 2004, A\&A, 418, 989

Patience, J., White, R. J., Ghez, A., et al. 2002, ApJ, 581, 684

Pfahl, E., \& Muterspaugh, M. 2006, ApJ, in press [arXiv:astro-ph/0606645]

Pichardo, B., Sparke, L. S., \& Aguilar, L. S. 2005, MNRAS, 359, 521

Pinfield, D. J., Jones, H. R. A., Lucas, P. W., et al. 2006, MNRAS, 368, 1281

Portegies Zwart, S. F., Hut, P., McMillan, S. L. W., \& Verbunt, F. 1997, A\&A, 328,143

Porto De Mello, G. F., \& Da Silva, L. 1997, ApJ, 476, L89

Poveda, A., \& Allen, C. 2004, RevMexAA, 21, 49

Raghavan, D., Henry, T. J., Mason, B. D., et al., ApJ, 646, 523 (R06)

Reffert, S., \& Quirrenbach, A. 2006, A\&A, 449, 699

Robinson, S. E., Laughlin, G., Bodenheimer, P., \& Fischer, D. 2006, ApJ, 643, 484

Rocha-Pinto, H. J., Castilho, B. V., \& Maciel, W. J. 2002, A\&A, 384, 912

Santos, N. C., Israelian, G., \& Mayor, M. 2004, A\&A, 415, 1153

Silvestri, N. M., Oswalt, T. D., Wood, M. A., et al. 2001, AJ, 121, 503

Sinachopoulos, D. 1988, A\&AS, 76, 189

Soderblom, D. R., \& Mayor, M. 1983, AJ, 105, 226

Takeda, G., \& Rasio, F. A. 2005, ApJ, 627, 1001

Takeda, G., Ford, E. B., Sills, A., et al. 2006, ApJS, submitted [arXiv:astro-ph/0607235]

Thebault, P., Marzari, F., Scholl, H., Turrini, D., \& Barbieri, M. 2004, A\&A, 427, 1097

Thebault, P., Marzari, F., \& Scholl, H. 2006, Icarus, 183, 193

Tokovinin, A. A., Griffin, R. F., Balega, Y. Y., Pluzhnik, E. A., \& Udry, S. 2000 Astron. Lett., 26, 116

Tokovinin, A. A., Thomas, S., Sterzik, M., \& Udry, S. 2006, A\&A, 450, 681

Tremaine, S., \& Zakamska, N. 2004, The Search for other worlds: Fourteenth Astrophysics Conference, AIP Conf. Proc., 713, 243

Udry, S., Mayor, M., \& Santos, N. C. 2003, A\&A, 407, 369

Valenti, J., \& Fischer, D. 2005, ApJS, 159, 141

Vogt, S. S., Butler, R. P., Marcy, G. W., et al. 2005, ApJ, 632, 638

Weinberg, M. D., Shapiro, S. L., \& Wasserman, I. 1987, ApJ, 312, 367

Wu, Y., \& Murray, N. 2003, ApJ, 589, 605

Zucker, S., \& Mazeh, T. 2002, ApJ, 568, L113 
S. Desidera and M. Barbieri: Properties of planets in binary systems, Online Material $p 1$

\section{Online Material}


Table 1. Properties of planets in multiple systems, of their host stars and their companions: planet $m \sin i$, period, semimajor axis, eccentricity, radial velocity semi-amplitude, metallicity and mass of the planet host (from B06); projected separation, semimajor axis and eccentricity (when available) and mass of the companions, and critical semiaxis for dynamical stability of planets. The asterisks in the last column mark systems discussed individually in Appendix C.

\begin{tabular}{|c|c|c|c|c|c|c|c|c|c|c|c|c|c|c|}
\hline $\begin{array}{l}\text { Object } \\
\text { HD }\end{array}$ & $\begin{array}{l}\text { Object } \\
\text { other }\end{array}$ & $\begin{array}{r}m \sin i \\
M_{\mathrm{J}}\end{array}$ & $\begin{array}{l}P \\
\mathrm{~d}\end{array}$ & $\begin{array}{r}a_{p} \\
\mathrm{AU}\end{array}$ & $e_{p}$ & $\begin{array}{r}K_{p} \\
\mathrm{~m} / \mathrm{s}\end{array}$ & {$[\mathrm{Fe} / \mathrm{H}]$} & $\begin{array}{r}M_{\text {star }} \\
M_{\odot}\end{array}$ & $\begin{array}{r}\rho \\
\mathrm{AU}\end{array}$ & $\begin{array}{l}a_{\text {bin }} \\
\mathrm{AU}\end{array}$ & $e_{\text {bin }}$ & $\begin{array}{r}M_{\text {comp }} \\
M_{\odot}\end{array}$ & $\begin{array}{c}a_{\text {crit }} \\
\mathrm{AU}\end{array}$ & Rem. \\
\hline 142 & & 1.31 & 350.3 & 1.045 & 0.26 & 33.9 & 0.100 & 1.24 & 138 & & & 0.56 & 35 & \\
\hline 1237 & GJ 3021 & 3.37 & 133.71 & 0.495 & 0.511 & 167.0 & 0.120 & 0.90 & 70 & & & 0.13 & 21 & \\
\hline 3651 & 54 Psc & 0.227 & 62.206 & 0.296 & 0.618 & 16.0 & 0.164 & 0.89 & 480 & & & 0.06 & 151 & * \\
\hline \multirow[t]{3}{*}{9826} & $v$ And & 0.687 & 4.617 & 0.0595 & 0.023 & 69.8 & 0.120 & 1.32 & 750 & & & 0.19 & 223 & \\
\hline & & 1.98 & 241.23 & 0.832 & 0.262 & 55.6 & 0.120 & 1.32 & 750 & & & 0.19 & 223 & \\
\hline & & 3.95 & 1290.1 & 2.54 & 0.258 & 63.4 & 0.153 & 1.32 & 750 & & & 0.19 & 223 & \\
\hline 11964 & & 0.61 & 2110.0 & 3.34 & 0.06 & 9.0 & 0.122 & 1.12 & 1379 & & & 0.67 & 325 & \\
\hline 13445 & GL 86 & 3.91 & 15.7649 & 0.113 & 0.0416 & 376.7 & -0.268 & 0.77 & & 18.4 & 0.40 & 0.49 & 3.1 & $a$ \\
\hline 16141 & 79 Cet & 0.26 & 75.523 & 0.363 & 0.252 & 11.99 & 0.170 & 1.12 & 223 & & & 0.29 & 62 & \\
\hline 19994 & 94 Cet & 1.69 & 535.7 & 1.428 & 0.30 & 36.2 & 0.186 & 1.35 & & 120 & 0.26 & 0.35 & 31 & $*$ \\
\hline 20782 & & 1.78 & 585.86 & 1.364 & 0.925 & 115.0 & -0.051 & 0.98 & 9080 & & & 0.84 & 1940 & \\
\hline 27442 & $\epsilon$ Ret & 1.56 & 428.1 & 1.27 & 0.06 & 32.2 & 0.420 & 1.49 & 251 & & & 0.60 & 62 & $a$ \\
\hline \multirow[t]{2}{*}{38529} & & 0.852 & 14.3093 & 0.1313 & 0.248 & 56.8 & 0.445 & 1.47 & 12000 & & & 0.50 & 3190 & $*$ \\
\hline & & 13.2 & 2165.0 & 3.74 & 0.3506 & 170.3 & 0.445 & 1.47 & 12000 & & & 0.50 & 3190 & \\
\hline 40979 & & 3.83 & 263.84 & 0.855 & 0.269 & 112.0 & 0.168 & 1.19 & 6400 & & & 0.75 & 1488 & \\
\hline 41004A & & 2.60 & 963.0 & 1.70 & 0.74 & 99.0 & 0.160 & 0.70 & 23 & & & 0.40 & 5.5 & * \\
\hline 41004B & & 18.4 & 1.3283 & 0.0177 & 0.081 & 6114.0 & 0.160 & 0.40 & 23 & & & 0.70 & 3.9 & $*$ \\
\hline 46375 & & 0.226 & 3.0235 & 0.0398 & 0.063 & 33.65 & 0.240 & 0.92 & 346 & & & 0.60 & 80 & \\
\hline 75289 & & 0.467 & 3.5092 & 0.0482 & 0.034 & 54.9 & 0.217 & 1.21 & 621 & & & 0.14 & 188 & \\
\hline \multirow[t]{4}{*}{75732} & $55 \mathrm{Cnc}$ & 0.0377 & 2.7955 & 0.0377 & 0.09 & 5.8 & 0.315 & 0.91 & 1062 & & & 0.26 & 291 & \\
\hline & & 0.833 & 14.652 & 0.1138 & 0.01 & 73.38 & 0.315 & 0.91 & 1062 & & & 0.26 & 291 & \\
\hline & & 0.157 & 44.36 & 0.238 & 0.071 & 9.6 & 0.315 & 0.91 & 1062 & & & 0.26 & 291 & \\
\hline & & 3.90 & 5552.0 & 5.97 & 0.091 & 47.5 & 0.315 & 0.91 & 1062 & & & 0.26 & 291 & \\
\hline 80606 & & 4.31 & 111.449 & 0.468 & 0.935 & 481.9 & 0.343 & 1.10 & 1200 & & & 0.90 & 260 & \\
\hline 89744 & & 8.58 & 256.80 & 0.934 & 0.677 & 267.3 & 0.265 & 1.64 & 2456 & & & 0.079 & 780 & \\
\hline 99492 & 83 Leo B & 0.109 & 17.0431 & 0.1232 & 0.254 & 9.8 & 0.362 & 0.86 & 515 & & & 1.01 & 100 & \\
\hline 109749 & & 0.277 & 5.23947 & 0.0629 & 0 & 28.58 & 0.250 & 1.21 & 490 & & & 0.78 & 113 & \\
\hline 114729 & & 0.95 & 1114.0 & 2.11 & 0.167 & 18.8 & -0.262 & 1.00 & 282 & & & 0.25 & 79 & \\
\hline 114762 & & 11.68 & 83.8881 & 0.363 & 0.336 & 615.2 & -0.653 & 0.89 & 130 & & & 0.07 & 40 & \\
\hline 120136 & $\tau$ Boo & 4.13 & 3.3125 & 0.048 & 0.023 & 461.1 & 0.234 & 1.35 & & 245 & 0.91 & 0.40 & 2.8 & $*$ \\
\hline 142022 & & 4.50 & 1928.0 & 2.93 & 0.53 & 92.0 & 0.190 & 0.90 & 820 & & & 0.60 & 188 & \\
\hline 147513 & & 1.18 & 528.4 & 1.31 & 0.26 & 29.3 & 0.089 & 1.07 & 4451 & & & 0.65 & 1044 & $a$ \\
\hline 178911 & & 7.35 & 71.511 & 0.345 & 0.139 & 346.9 & 0.285 & 1.06 & 640 & & & 1.89 & 108 & $b$ \\
\hline 186427 & 16 CygB & 1.68 & 798.5 & 1.681 & 0.681 & 50.5 & 0.038 & 0.99 & 850 & & & 1.19 & 164 & $b$ \\
\hline 188015 & & 1.50 & 461.2 & 1.203 & 0.137 & 37.6 & 0.289 & 1.09 & 684 & & & 0.21 & 198 & \\
\hline 189733 & & 1.15 & 2.219 & 0.0312 & 0 & 205.0 & -0.030 & 0.82 & 216 & & & 0.19 & 61 & $*$ \\
\hline \multirow[t]{2}{*}{190360} & GJ $777 \mathrm{~A}$ & 0.0587 & 17.1 & 0.1303 & 0.01 & 4.6 & 0.213 & 1.01 & 3000 & & & 0.20 & 864 & \\
\hline & & 1.55 & 2891.0 & 3.99 & 0.36 & 23.5 & 0.213 & 1.01 & 3000 & & & 0.20 & 864 & \\
\hline 195019 & & 3.69 & 18.2013 & 0.1388 & 0.0138 & 271.5 & 0.068 & 1.07 & 150 & & & 0.70 & 35 & \\
\hline 196050 & & 2.90 & 1378.0 & 2.454 & 0.228 & 49.7 & 0.229 & 1.15 & 511 & & & 0.36 & 138 & \\
\hline 213240 & & 4.72 & 882.7 & 1.92 & 0.421 & 96.6 & 0.139 & 1.22 & 3898 & & & 0.15 & 1177 & \\
\hline 222404 & $\gamma$ Cep & 1.77 & 905.0 & 2.14 & 0.12 & 27.5 & 0.180 & 1.59 & & 18.5 & 0.36 & 0.40 & 4.0 & \\
\hline 222582 & & 7.75 & 572.38 & 1.347 & 0.725 & 276.3 & -0.029 & 0.99 & 4746 & & & 0.36 & 1246 & \\
\hline
\end{tabular}

Binary references: HD 142: R06; HD 1237: Chauvin et al. (2006); HD 3651: Mugrauer et al. (2006b); HD 9826: Lowrance et al. (2002), E04; HD 11964: R06; HD 13445: Lagrange et al. (2006), Mugrauer \& Neuhauser (2005); HD 16141: Mugrauer et al. (2005); HD 19994: Hale (1994), E04; HD 20782: this paper; HD 27442: Chauvin et al. (2006), R06; HD 38529: R06; HD 40979: E04; HD 41404: E04; HD 46375: Mugrauer et al. (2006a); HD 75289: Mugrauer et al. (2004b); HD 75732: E04, Mugrauer et al. (2006a); HD 80606: E04; HD 89744: Mugrauer et al. (2004a); HD 99492: R06; HD 109749: this paper; HD 114729: Mugrauer et al. (2005); HD 114762: Patience et al. (2002), E04; HD 120136: Hale (1994), E04; HD 142022: Eggenberger et al. (2006b); HD 147513: Mayor et al. (2004), R06, Silvestri et al. (2001); HD 178911: Tokovinin et al. (2000), E04; HD 186427: Hauser \& Marcy (1999), Patience et al. (2002), E04; HD 188015: R06; HD 189733: Bakos et al. (2006a); HD 190360: E04; HD 195019: E04; HD 196050: Mugrauer et al. (2005); HD 213240: Mugrauer et al. (2005); HD 222404: Hatzes et al. (2003); HD 222582: R06.

a The secondary is a white dwarf; the original mass was larger and the separation smaller. See Appendix B for details.

${ }^{b}$ Hierarchical triple system, the sum of the masses of the two companions is listed here. See Appendix C for details. 


\section{Appendix A: Binaries among new planet hosts}

As planet announcements arise quite frequently, existing compilations quickly become incomplete. Twenty-one stars are not considered in the latest study of multiplicity of planet hosts (R06). Most of them are new planet hosts while some are stars with companions with mass between 13 to $24 M_{\mathrm{J}}$, not considered by R06 but included in B06 catalog.

We searched for companions of these stars in existing astronomical catalogs such as Hipparcos (ESA 1997), CCDM (Dommaget \& Nys 2002), WDS (Mason et al. 2001), and ADS (Aitken 1932). For the following 16 stars there are no evidence of binarity in the literature: HD 4308, HIP 14810, HD 33283, HD 66428, HD 81040, HD 86081, HD 99109, HD 102195, HD 107148, HD 109143, HD 118203, GJ 581, HD 137510, HD 187085, HD 212301, HD 224693.

HD 33564 and HD 164922 are included in CCDM and WDS (the latter also in ADS) as triple systems, but the companions display different proper motion to the planet hosts and thus they are not physical companions. Two additional objects (HD 20782 and HD 109749) are binaries. The physical association of the companions and their physical properties are discussed in the next subsections. Finally, the binarity of HD 189733 is discussed in a dedicated paper by Bakos et al. (2006a).

\section{A.1. HD 20782}

HD 20782 is listed as a binary in the CCDM catalog (CCDM 03201-2850). The companion is HD 20781. The very large separation, 252 arcsec, that corresponds to $9080 \mathrm{AU}$ at the distance of HD 20782 (36 pc) makes a detailed check of physical association mandatory. Table A.1 lists the stellar parameters of the two stars. Hipparcos parallaxes and proper motions, and Nordstrom et al. (2004) radial velocities, are fully compatible within errors for the two components. This strongly suggests physical association. Therefore, HD 20782 and HD 20781 form a very wide common proper motion pair. Considering the nominal radial velocity and proper motion differences, the pair could be bound. The observed separation is very large but not extreme for binaries in the solar neighborhood (Poveda \& Allen 2004). Clearly, the orbit of the wide binary remains unconstrained.

We tested the dynamical stability of the system via numerical integration and we have performed 10 simulation of this system following the evolution for $200 \mathrm{Myr}$ (577 binary orbits) using a RADAU integrator (Everhart et al. 1985). The binary orbit was assumed with typical values of $a_{\text {bin }}=6000 \mathrm{AU}$ and $e_{\text {bin }}=0.60$, the orbital parameter of the planet was chosen equal to the observed values, and inclination was chosen slightly inclined $\left(i=5^{\circ}\right)$ with respect to the binary orbital plane. The planet results stable over all the simulation, thus we argue that the large binary separation ensures dynamical stability of the planet in spite of its very high eccentricity.

Only two extrasolar planets have orbits with eccentricities larger than 0.8: HD 20782b and HD 80606b. Both their host stars are member of wide common proper motion pairs.

While the link between the high eccentricity and binarity still needs statistical confirmation, nevertheless it is interesting to investigate the possible ways in which a distant companion might have induced extreme planet eccentricities.

As discussed in Sect. 4, the extremely high eccentricity of HD 20782b is unlikely to be due to the Kozai mechanism, as the period of the eccentricity oscillation is much larger than the Hubble time.
Table A.1. Stellar properties of HD 20782 and HD 20781.

\begin{tabular}{|c|c|c|c|}
\hline Parameter & HD 20782 & HD 20781 & Ref. \\
\hline$\mu_{\alpha}(\mathrm{mas} / \mathrm{yr})$ & $348.88 \pm 0.50$ & $349.07 \pm 0.78$ & 1 \\
\hline$\mu_{\delta}$ (mas/yr) & $-64.82 \pm 0.73$ & $-67.80 \pm 1.00$ & 1 \\
\hline$R V\left(\mathrm{~km} \mathrm{~s}^{-1}\right)$ & $39.5 \pm 0.2$ & $39.6 \pm 0.2$ & 2 \\
\hline$\pi$ (mas) & $27.76 \pm 0.88$ & $27.86 \pm 1.23$ & 1 \\
\hline$R_{\min }(\mathrm{kpc})$ & \multicolumn{2}{|c|}{4.95} & 2 \\
\hline$R_{\max }(\mathrm{kpc})$ & \multicolumn{2}{|c|}{8.16} & 2 \\
\hline$e c c$ & \multicolumn{2}{|c|}{0.24} & 2 \\
\hline$z_{\max }(\mathrm{kpc})$ & \multicolumn{2}{|c|}{0.08} & 2 \\
\hline$V$ & 7.366 & 8.457 & 2 \\
\hline ST & G3V & K0V & 1 \\
\hline \multirow[t]{3}{*}{$\operatorname{Mass}\left(M_{\odot}\right)$} & 0.90 & 0.84 & 2 \\
\hline & 1.00 & & 3 \\
\hline & $0.969^{+0.024}$ & & 4 \\
\hline \multirow[t]{3}{*}{$\operatorname{Age}_{\text {isoc }}(\mathrm{Gyr})$} & $13.0^{-0.022}$ & & 2 \\
\hline & $7.1^{+1.9}$ & & 3 \\
\hline & $9.68 \pm 1.76$ & & 4 \\
\hline $\mathrm{Age}_{H K}(\mathrm{Gyr})$ & $3-6$ & 6 & 5,6 \\
\hline
\end{tabular}

References: 1. Hipparcos (ESA 1997); 2. Nordstrom et al. (2004); 3. Valenti \& Fischer (2005); 4. Takeda et al. (2006); 5. Jones et al. (2006); 6. Gray et al. (2006).

An interesting possibility to explain both the very large separation of the binary and the very high eccentricity of the planet orbiting the primary is a dynamical encounter of the binary (or of an originally higher multiplicity system) within a star cluster or with a passing star, that might have perturbed both the binary and the planet orbit. According to the simulations by Weinberg et al. (1987), the probability of survival for a binary with an initial semimajor axis of about 10000 AU after $4 \mathrm{Gyr}$ is less than $50 \%$. Furthermore, the actual semimajor axis is probably larger than the observed projected separation by about 30\% (Duquennoy \& Mayor 1991; Fischer \& Marcy 1992), further decreasing the probability of survival, if this is the case of HD 20782. The galactic orbit of HD 20782 (Table A.1) makes the star on average closer to the Galactic center than the Sun, further increasing the chance of stellar encounters, because of the higher stellar density. Therefore, at the age of the system $\left(7.1_{-4.1}^{+1.9}\right.$ Gyr; Valenti \& Fischer 2005; $9.68 \pm 1.76$; Takeda et al. 2006), the probability that the currently observed orbit is not the original one but it was significantly modified by dynamical encounters in the Galactic disk is rather high.

The process of destruction of wide binaries is typically the result of a large number of weak encounters (see e.g. Portegies Zwart et al. 1997). The changes in the binary orbit caused by one or more stellar encounters might alter the orbit of the planet(s) orbiting one of the components. A multi-planet system can be destabilized, leading to chaotic evolution of the planetary orbits. Dedicated dynamical modelling should be performed to check if the current configuration of the system might represent a possible outcome of a stellar encounter. This is postponed to future work. The hypothesis of stellar encounters appears less appealing to explain the extremely high eccentricity of HD $80606 \mathrm{~b}$, as in this case the projected separation of the binary is about $1200 \mathrm{AU}, 7-8$ times smaller than for HD 20782. However, the real orbit remains unknown.

The possibility that the high planet eccentricity is unrelated to the presence of the stellar companions and is caused by internal evolution of the planetary system cannot be excluded. Conclusive inferences on the origin of the very high eccentricity of HD 20782b and HD 86606b are not possible from the 
Table A.2. Stellar properties of HD 109749 and HD 109749B.

\begin{tabular}{lccc}
\hline \hline Parameter & HD 109749 & HD 109749B & Ref. \\
\hline$\mu_{\alpha}(\mathrm{mas} / \mathrm{yr})$ & $-157.89 \pm 1.41$ & -158 & 1,2 \\
$\mu_{\delta}(\mathrm{mas} / \mathrm{yr})$ & $-5.48 \pm 1.25$ & -6 & 1,2 \\
$\pi$ (mas) & $16.94 \pm 1.91$ & & 1 \\
$V$ & 8.09 & 10.76 & 4,5 \\
$J_{2 \text { MASS }}$ & $7.057 \pm 0.021$ & $8.788 \pm 0.024$ & 6 \\
$H_{2 \text { MASS }}$ & $6.797 \pm 0.031$ & $8.289 \pm 0.051$ & 6 \\
$K_{2 \text { MASS }}$ & $6.678 \pm 0.024$ & $8.123 \pm 0.024$ & 6 \\
$M_{V}$ & $4.23 \pm 0.25$ & $6.90 \pm 0.25$ & 1,5 \\
Mass $\left(M_{\odot}\right)$ & 1.11 & 0.78 & 5 \\
Mass $\left(M_{\odot}\right)$ & 1.2 & & 4
\end{tabular}

References: 1. Hipparcos (ESA 1997); 2. USNO-B1.0 Catalog (Monet et al. 2003) 3. Nordstrom et al. (2004); 4. Fischer et al. (2006); 5. This paper; 6. 2MASS (Cutri et al. 2003).

Table A.3. Relative astrometry of HD 109749.

\begin{tabular}{lccl}
\hline \hline Epoch & $\rho$ & $\theta$ & Ref. \\
\hline 1881 & 8.2 & 179 & CCDM \\
1987.35 & $8.35 \pm 0.04$ & $179.76 \pm 0.21$ & Sinachopoulos (1988) \\
1991.25 & $8.353 \pm 0.014$ & 180.0 & Hipparcos \\
1999.27 & 8.28 & 179.75 & 2MASS \\
\hline
\end{tabular}

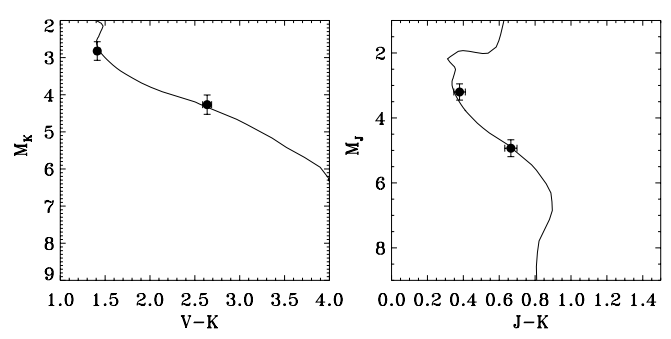

Fig. A.1. Absolute magnitudes and colors for the components of HD 109749 (filled circles). Left panel: $M_{K}$ vs. $V-K$; right panel: $M_{\mathrm{J}}$ vs. $J-K$. The $4 \mathrm{Gyr}, Z=0.030$ isochrone by Girardi et al. (2002) is overplotted.

available data. Further clues will come from follow-up observations, such as the search for further planetary and stellar companions, and from the discovery of further planets with very highly eccentric orbit and the study of the binarity of their hosts.

\section{A.2. $H D 109749$}

HD 109749 is listed in Hipparcos and CCDM catalogs as a binary (CCDM J12373-4049). The Hipparcos magnitude of the secondary transformed to the standard system following Bessell (2000) yields $V=10.76$, about 2.5 mag fainter than the primary ( $V=8.09$, Fischer et al. 2006; see Table A.2). Table A.3 shows the relative astrometry for the two components. The constancy of the projected separation and position angle, coupled with the rather large proper motion of HD 109749, makes the physical association between the two objects very probable. The projected separation of 8.35 arcsec corresponds to about $500 \mathrm{AU}$.

To further check the physical association between the two stars, we identified them in 2MASS catalog (Cutri et al. 2003). The photometry is shown in Table A.2. When placed in a color-magnitude diagram, both components lies along the same isochrone, indicating a common distance and confirming the physical association (Fig. A.1). The masses derived from the isochrone shown in Fig. A. 1 are 1.11 and $0.78 M_{\odot}$.

\section{Appendix B: White dwarf companions of planet hosts}

Three planets are orbiting stars with white dwarfs companions. For binaries wide enough to escape common envelope evolution, the mass loss from the originally more massive star during the RGB and AGB phases determines a widening of the orbit. The mass loss phase lasts several Myr, longer than the typical binary orbital periods. In these conditions, the widening of the binary orbit can be approximated by $a_{\text {end }}=a_{\text {start }} M_{\text {start }} / M_{\text {end }}$, and no eccentricity changes are expected (Lagrange et al. 2006 and references therein). In this section, we consider the three planet-host binaries with a WD, placing constraints on the original configuration of the system in terms of binary separation and mass ratio.

\section{B.1. $H D 13445=G L 86$}

The companion of G186 was discovered by Els et al. (2001) and it was classified as a brown dwarf on the basis of the nearinfrared colors. Mugrauer \& Neuhauser (2005) showed that the companion is instead a white dwarf. Lagrange et al. (2006) further constrained the properties of the system, using archive radial velocity and new photometry and position measurements ${ }^{6}$. However, they also noted some inconsistency between the white dwarf cooling age (1.2-1.8 Gyr depending on white dwarf mass and atmosphere composition), the age of the star as derived from chromospheric activity (about 2-3 Gyr), and the presence of the planet. In fact, such a young age implies an original mass of the companion that is too large to be compatible with a dynamically stable orbit for the planet once the widening of the binary orbit due to mass loss from the originally more massive star is taken into account.

A picture that might explain the properties of the system is the following. Kinematic parameters $\left(U=-102 \mathrm{~km} \mathrm{~s}^{-1}\right.$, $V=-75 \mathrm{~km} \mathrm{~s}^{-1}, W=-29 \mathrm{~km} \mathrm{~s}^{-1}$, galactic orbit $R_{\min }=4.0 \mathrm{kpc}$, $R_{\max }=9.2 \mathrm{kpc}, e=0.39, Z_{\max }=0.4 \mathrm{kpc} ;$ Nordstrom et al. 2004) indicate a very old age ${ }^{7}$. The kinematic criterium of Bensby et al. (2005) suggests a probable membership to the thick disk. Chemical abundances can also be used to constrain the galactic population. At the metallicity of GL86 $([\mathrm{Fe} / \mathrm{H}] \sim-0.20)$, differences between thin and thick stars are fairly small (the typical $[\alpha / \mathrm{Fe}]$ of thin disk and thick stars is about +0.05 and +0.15 respectively, Bensby et al. 2005). High-resolution abundances analysis of several elements were published by Allende Prieto et al. (2004), Valenti \& Fischer (2005), Santos et al. (2004), and Gilli et al. (2006). The uncertainty in the chemical abundances of GL 86 and the discrepancies between different studies do not allow a firm classification, but they appear compatible with thick disk membership or with thick disk-thin disk transition

\footnotetext{
6 The available observational constraints did not allow Lagrange et al. (2006) to derive a unique solution for the binary orbit. The orbit listed in Table 1 is a plausible, representative solution.

7 The difference between the kinematic and activity age of GL 86 was previously noted by Rocha Pinto et al. (2002).
} 
objects $^{8}$. Thick disk stars with this metallicity have typical ages of $8 \mathrm{Gyr}$ according to Bensby et al. (2005). This is compatible with the lower limit ( $68 \%$ confidence level) of $8.5 \mathrm{Gyr}$ estimated by Takeda et al. (2006) from isochrone fitting. If GL 86 is instead a thin disk star, its kinematic parameters strongly supports an old age.

Matching the cooling age of the white dwarf given by Lagrange et al. (2006) with the assumed age of about 8 Gyr for the system requires an original mass of about $1.2 M_{\odot}$. Older ages yield lower initial masses.

A $1.2 M_{\odot}$ star ends its life as a $\sim 0.54 M_{\odot}$ white dwarf. The amount of mass loss implies a widening of the binary orbit of about $40-50 \%$ of its original separation. To match the semimajor axis of 18.4 AU derived by Lagrange et al. (2006), the semimajor axis of the original orbit had to be about $13 \mathrm{AU}$.

A fraction of the mass loss by GL86 B during the RGB and AGB evolution should have been captured by the planet host. A rough estimate based on the BSE binary evolution code by Hurley et al. (2002), using the default wind and accretion parameters, is $0.017 M_{\odot}$. The $\sim 0.017 M_{\odot}$ accreted mass corresponds to roughly half of the mass of the convective envelope of GL86 $\left(M_{\mathrm{ce}}=0.039 \pm 0.003\right.$; Takeda et al. 2006). The lack of peculiar abundance of neutron capture elements $([\mathrm{Ba} / \mathrm{Fe}]=-0.12$; Allende Prieto et al. 2004) suggests an original mass for the AGB star smaller than 1.3-1.5 $M_{\odot}$ (Busso et al. 1999), in agreement with our estimate of $\sim 1.2 M_{\odot}$.

Along with mass, angular momentum should also have been transferred to the planet host. This was invoked to explain the high rotation rates of companions of hot white dwarfs such as the barium dwarf 2RE J0357+283 (Jeffries \& Smalley 1996). The accretion of angular momentum by GL86 would explain its activity level typical of a much younger star.

This scenario would be able to explain the available observational constraints, including the stability of the planetary orbit. However, the formation of a planet with a companion at about $13 \mathrm{AU}$ (and with an eccentricity of 0.4 ) represents a challenge for current models (Hatzes \& Wuchterl 2005). The signature of accretion of angular momentum by GL86 is an indication that the binary system was in its current status at the time of the mass loss by GL86B, about 1.5 Gyr ago. Earlier modifications of the binary orbit due to e.g. dynamical interactions within the native star cluster (Pfahl \& Muterspaugh 2006) cannot be excluded. The possible effects of the mass and angular momentum accretion on the mass and orbit of the planet remain to be investigated.

\section{B.2. $H D 27442=\epsilon$ Ret}

HD $27442=\epsilon$ Ret has a companion at a projected separation of about 13 arcsec $=240$ AU. According to Chauvin et al. (2006), visual and near-infrared photometry are not compatible with any main sequence star. They then argue that the companion is a white dwarf. The mass of the planet host yields a lower limit to the original mass of the companion. Valenti \& Fischer (2005) determine a mass of $1.49 M_{\odot}$ (the value also adopted by B06), with an age of $3.5 \mathrm{Gyr}$, while Takeda et al. (2006)

\footnotetext{
${ }^{8}$ The relative abundances of Allende Prieto et al. (2004) $([\mathrm{O} / \mathrm{Fe}]=+0.21,[\mathrm{Mg} / \mathrm{Fe}]=+0.13,[\mathrm{Si} / \mathrm{Fe}]=+0.08,[\mathrm{Ca} / \mathrm{Fe}]=+0.18$, $[\mathrm{Ti} / \mathrm{Fe}]=+0.11,[\mathrm{Eu} / \mathrm{Fe}]=+0.30)$ fit fairly well the expected abundance pattern for a thick star of the metallicity of Gl86, those by Gilli et al. (2006) and Ecuvillon et al. (2006) are more ambiguous $([\mathrm{O} / \mathrm{Fe}]=-0.01,[\mathrm{Mg} / \mathrm{Fe}]=+0.21,[\mathrm{Si} / \mathrm{Fe}]=-0.02,[\mathrm{Ca} / \mathrm{Fe}]=-0.10$, $[\mathrm{Ti} / \mathrm{Fe}]=+0.19)$. Valenti \& Fischer $(2005)$ find an abundance ratio similar to that of Allende Prieto et al. (2004) for two elements $([\mathrm{Si} / \mathrm{Fe}]=+0.11,[\mathrm{Ti} / \mathrm{Fe}]=+0.10)$.
}

list $M=1.48_{-0.08}^{+0.22} M_{\odot}$ with an age of $2.84_{-0.36}^{+0.60}$ Gyr. A lower limit to the WD mass estimated using the BSE code (taking into account the high metallicity of $\epsilon \operatorname{Ret} ;[\mathrm{Fe} / \mathrm{H}]=+0.42)$ is $M \sim 0.60 M_{\odot}$. Theoretical models should be taken with caution at these extremely high metallicities, as they fail to explain the WD luminosity function of the old super-metal-rich open cluster NGC 6791 (Bedin et al. 2005). The relatively wide separation from the primary (13 arcsec) and the moderately bright magnitude ( $V \sim 12.5$, WDS) open the perspective for a more detailed characterization of $\epsilon$ Ret B. We can guess that the system had lost more than one third of its original mass, implying a substantial widening of the binary orbit. The original separation might have been about $150 \mathrm{AU}$, making the influence of the companion not negligible.

\section{B.3. $H D 147513$}

HD 147413 was classified as a member of the Ursa Major moving group by Soderblom \& Mayor (1983) while King et al. (2003) classified the membership as unprobable, on the basis of the discrepant kinematic parameters. The activity level and the position on the color-magnitude diagram are instead fully compatible with membership. Its companion (WD 1620-39= HIP 80300) is a widely studied WD, for which Silvestri et al. (2001) derived a mass of $0.65 \pm 0.01 M_{\odot}$ and a cooling age of $20 \mathrm{Myr}$. The planet host was claimed to be a barium star $([\mathrm{Ba} / \mathrm{Fe}]=+0.37)$ by Porto De Mello \& Da Silva (1997) but Castro et al. (1999) showed that the high barium abundance is shared by other stars in the UMa moving group (and possibly by other young stars). This points toward a primordial abundance and not to accretion by the WD companion, unexpected on the basis of the large separation (more than $4000 \mathrm{AU}$ ).

We estimated the possible original mass of the (now) WD companion using the BSE code (Hurley et al. 2002). Adopting the most recent age estimate of the UMa moving group (500 Myr; King et al. 2003) and the cooling age by Silvestri et al. (2001) leads to an original mass of 3.0 $M_{\odot}$ and a WD mass of $0.75 M_{\odot}$, some $0.1 M_{\odot}$ larger than that derived by Silvestri et al. (2001). The WD mass and cooling age by Silvestri et al. (2001) can be matched simultaneously by adopting an initial mass of $2.3 M_{\odot}$ and a system age of $1 \mathrm{Gyr}$. This age is still marginally compatible with the photometry and activity level, if the star is not a member of UMa moving group. We conclude that the original separation of the binary was about a half of the present one, as the system has lost about half of its original mass. Considering the current very wide separation, this does not change the classification of this planet host as a very wide binary.

\section{Appendix C: Individual objects}

We further discuss individual objects.

- HD 3651: a brown dwarf companion was recently identified by Mugrauer et al. (2006b). Its mass depends largely on the age of the system (20-60 $M_{\mathrm{J}}$ for ages of $1-10 \mathrm{Gyr}$ respectively). Takeda et al. (2006) favor a very old age (older than $11 \mathrm{Gyr}$ at a $68 \%$ confidence level). We then assume $M=0.06 M_{\odot}$. Such a mass coupled with the wide separation (480 AU) indicates that the classification of this object within the binary companions and not within the "super-planets" is appropriate. The critical semiaxis for this system is uncertain as Eq. (1) of Holman \& Wiegert (1999) is not defined for such an extreme mass ratio. 
- HD 19994: the visual orbit by Hale (1994) is very preliminary.

- HD 38529: astrometric acceleration detected by Hipparcos. According to Reffert \& Quirrenbach (2006), this acceleration is due to the outer "planet" that has $m=37_{-19}^{+36} M_{\mathrm{J}}$. The classification of this star as a two-planet host with a wide stellar companion or a single-planet host with a brown dwarf and a further wide companion is then ambiguous.

- HD 41004: the stellar metallicity of HD 41004B is not listed by B06. We assume that of the primary.

- HD 114762: a companion with mass $0.075 M_{\odot}$ was found by Patience et al. (2002). Chauvin et al. (2006) report a candidate companion, whose physical association has to be established.

$-\tau$ Boo: the very eccentric orbit by Hale (1994: $e=0.91$ ) implies a periastron of about 20 AU. However, the latest position measurement by Patience et al. (2002) indicates a larger separation and similar position angle than predicted by the preliminary orbit. A redetermination of the binary orbit, taking into account also the long term radial velocity trend reported by B06, would be useful. A further L dwarf companion candidate at large separation has been reported by Pinfield et al. (2006). The physical association has to be confirmed.

- HD 178911: hierarchical triple system; a tight pair (masses 1.10 and $0.79 M_{\odot}$, semimajor axis $3 \mathrm{AU}$; Tokovinin et al. 2000) is at a projected separation of about $640 \mathrm{AU}$ from the planet host.

- HD $186427=16$ Cyg: hierarchical triple system; 16 Cyg A (the companion of the planet host, at a projected separation of $850 \mathrm{AU}$ ) was shown to be a binary with a projected separation of about $70 \mathrm{AU}$ (Patience et al. 2002). The masses of $16 \mathrm{Cyg} \mathrm{A}$ and its companion are about 1.02 and $0.17 M_{\odot}$ respectively.

- HD 189733: this is the only host of a transiting planet in a binary system. The plane of the planet orbit is thus known. According to Bakos et al. (2006a), from the available radial velocity and position data of the binary system, there is evidence that the binary and planetary orbit are not coplanar. Therefore, this object is a candidate for the occurrence of Kozai migration. Characterization of the binary orbit will allow to test this scenario.

\section{C.1. Objects not included in B06 catalog}

The criteria for the inclusion of planets in the "Catalog of Nearby Exoplanets" are different to other compilations such as the Extrasolar Planet Encyclopedia maintained by J. Schneider ${ }^{9}$. Three stars classified as planet hosts by the Extrasolar Planet Encyclopedia and not included in the Catalog of Nearby Exoplanets are members of multiple systems:

- HD 188753A was announced to host a hot Jupiter by Konacki (2005b). This star is a member of a hierarchical triple. The close pair HD $188753 \mathrm{BC}$ reaches at periastron a distance of just 6 AU from HD 188753A. If confirmed, this candidate would be the planet in the closest multiple system found up to now.

- HD 196885: a planet candidate was reported in 2004 at http://exoplanets.org/esp/hd196885/ hd196885. shtml, without successive publication of further details. The star was found to be a tight binary $(0.7$ arcsec, 25 AU projected separation) by Chauvin et al. (2006).

- HD 219449 is a giant star. It was included in the study of R06 that showed that the system is a hierarchical triple. A close pair, HD 219430 (separation $18 \mathrm{AU}$ ) is at $2250 \mathrm{AU}$ projected separation from the primary (the planet host candidate).

\section{C.2. Additional unconfirmed binaries}

A few additional planet hosts not included in Table 1 have companion candidates whose identification or physical association needs confirmation: These are:

- HD 169830: a candidate companion at 11 arcsec was shown by R06 to have photometric distance compatible with physical association with the planet host. However, the low proper motion of the primary does not allow confirmation. Further observations (radial velocity, spectral classification) are required.

- HD 217107: it is listed in WDS as a binary with a companion with a projected separation of $0.3-0.5 \operatorname{arcsec}=6-10 \mathrm{AU}$. The magnitude difference is not provided. According to R06, this detection needs confirmation. Chauvin et al. (2006) reported null results of their adaptive optics search using NACO. Their limit on $\Delta K_{\mathrm{S}}$ at $0.3 \mathrm{arcsec}$ is about $7.5 \mathrm{mag}$, corresponding to a mass of about $0.1 M_{\odot}$. Vogt et al. (2005) also report non detection of stellar companions at separations larger than 0.1 arcsec. The Hipparcos catalog does not include evidence for astrometric motion, expected for a stellar companion at $6 \mathrm{AU}$. As noted by R06, the presence of a stellar companion at 6 AU would not be dynamically compatible with that of the outer planet ( $a \sim 4$ AU). One possible explanation is that the system is seen nearly pole-on and that the stellar companion and the outer "planet" are the same object. However, this possibility is not convincing considering that the second epoch observation listed in WDS is 1997 , fairly close to the periastron of the RV orbit $(1998.7 \pm 0.3)$. Detection should have been easier in the past few years, in contrast to the null detections by deep adaptiveoptics searches.

- HD 168443: Reffert \& Quirrenbach (2006) derived an astrometric mass of $36 \pm 12 M_{\mathrm{J}}$ for the outer "super-planet" HD 168443 c. As for HD 38529, there is then ambiguity about the classification of the object.

- HD 111232 and HD 150706: astrometric acceleration detected by Hipparcos (see R06).

- HD 52265, HD 121504, HD 141937, HD 154857, HD 162020, HD 179949 and HD 183263: first epoch observations by Chauvin et al. (2006) revealed companion candidates. Follow-up observations are required to establish their physical association with the planet hosts.

\footnotetext{
${ }^{9}$ www. exoplanet.eu
} 